\title{
Zur Kinetik der zellfreien Gärung.
}

\author{
Von \\ Otto Meyerhor.
}

(Mit 7 Kurvenzeichnungen im Text.)

(Aus dem physiologischen Institut in Kiel.)

(Der Redaktion zugegangen am 26. April 1918.)

Die Anwendung der Gesetze der chemischen Reaktionskinetik stößt bekanntlich schon gegenüber den einfacheren enzymatischen Vorgängen auf große Hindernisse, die häufig mehr durch gewaltsame Interpretation des Tatsachenmaterials - um es dem Massenwirkungsgesetz gefügig zu machen als durch wirkliche Aufklärung des Reaktionsmechanismus aus dem Wege geräumt werden: Wenn auch die in einem erheblichen Konzentrationsbereich des Substrats beobachtete Konstanz der Reaktionsgeschwindigkeit mit gutem Grund meist auf die Bildung eines Zwischenprodukts von Enzym und Substrat zurückgeführt wird, das unter der Bindung der ganzen Enzymmenge langsamer zerfällt als sich bildet, ${ }^{1}$ ) so ist doch mit dieser Erklärung weder das experimentelle Material erschöpft, noch auch mehr als eine ganz allgemeine Vorstellung über den Mechanismus gewonnen. Diese Schwierigkeiten wachsen nun noch außerordentlich an, wenn wir einen an sich so verwickelten Vorgang wie die alkoholische Gärung in vitro betrachten, die auf einem Ineinanderspielen einer ganzen Reihe von Enzymen und teils bekannten, teils unbekannten Zwischenstufen des Zuckerzerfalls beruht. Das von Buchner und seinen Mitarbeitern angesammelte große Tatsachenmaterial über die zellfreie Gärung ist im allgemeinen für eine nachträgliche Analyse in dieser Richtung schlecht verwertbar,

1) Adrian Brown, Journ. Chem. Soc. Bd. 81, S. 373 (1902).

Hoppe-Seyler's Zeitschrift f. physiol. Chemie. CII. 
einmal weil sehr wichtige Faktoren des Gärungsablaufs erst allmählich bekannt geworden sind, und dann weil die von ihm benutyte, später vielfach festgehaltene Methode, die Wägung des Kohlensäureverlusts in großen Zeiträumen, solchen Betrachtungen nicht günstig ist. Erst als die englischen Forscher Harden und Young zu Methoden übergingen, die Messungan in ganz kurzen Zeiträumen ( 5 Minuten) gestatteten, eröffnete sich die Möglichkeit, in den Reaktionsmechanismus tiefer einzudringen. Für solche Untersuchungen kommt es weniger auf die Gesamtleistung einer bestimmten Menge Hefesaft an von Buchner als «Gärkraft, des Saftes definiert - als auf die Geschwindigkeit der Reaktion unter variabeln Umständen, und zwar hauptsächlich in den ersten Versuchsstunden, wo die Intaktheit aller an der Gärung unmittelbar beteiligten Enzyme vorausgesetzt werden darf.

Meine Versuche schließen sich zu einem großen Teil an die Arbeiten von Harden und Young an und an die Betrachtungen, die der erstere in dem Kapitel sthe Mechanism of Fermentation, seines Buches: Alcoholic Fermentation (London 1911) über den Reaktionsverlauf angestellt hat. Dabei ergaben sich einige neue Tatsachen, die die Vorstellungen der englischen Forscher zu ergänzen und in Einzelheiten zu modifizieren imstande sind, wenn sie auch das Wesentliche derselben bestätigen. In diesem Zusammenhang wurden auch Versuche über die Rolle des Coferments bei der Gärung ausgeführt. Daß diese verhältnismäßig am wenigsten gefördert werden konnten, hat seinen Grund in der zunehmend schlechteren Beschaffenheit der Hefe zur Gewinnung gärkräftiger und haltbarer Macerationssäfte, die wohl nur mit der Kriegsernährung der Hefe zusammenhängen kann. Gerade für solche Studien ist nach dem von mir benutzten Verfahren ein mehrstündiges Waschen des Safts über dem Ultrafilter zur Entfernung des Koferments erforderlich; dabei macht sich die schlechte Haltbarkeit der Zymase besonders geltend. Natürlich ist auch für die kinetischen Experimente eine gute und vor allem gleichmäßige Beschaffenheit des Safts Voraussetzung. Dieselbe.konnte auch, wenn die Empfindlichkeit gegenüber 
Zucker-, Phosphatkonzentration und Verdlinnung ausprobiert war, für die zu den Vorsuchon dienendon Prïparato gewălirleistet werden.

Ein anderer Teil der Arbeit diento dor Prüfung dor Frage, ob die für die Hefesafteällungen kürzlich gewonnena Erkenntnis, daß es sich dabei um durch Narkotika sonsibilisierto Salzfällungon handell, ${ }^{1}$ ) much aul die narkotische Hemmung anwendbar ist, nachdem Warburg und Wiose $l^{2}$ ) früher auf den Parallelismus von Füllung und Hommung aufmerksam gemacht und darauf oine Thoorio der Wirkung der Narkotika aufgobaut haben. Gleichzeitig wurdun die Gärringshemmungen an 'Irockenhofe gemessen.

Für dio Studien zur Gïrungskinotik bietot dio Benutzung dos Macorationssafts (Lobodow)") gegenuber dom Heleprolissaft den großen Vortoil des völligen Mangels au Sellıstghtrung, dio im Preßsaft gerndo flir dicso Zwocko eine starke Kompllkation bedeutet und manche Erscheinungen vorschleiort. Datnebon ist noch bei guton und dauerbafton 'Trockonhefen dle grofo Gileichmäßigkoil des Safts zu rïhımon, der für Monale konstante Bigenschafton bosilat - cin Vorzug, der allerdings bei don hier milgetoilton Vorsuchen in Wogfall kim. Sio wurdon \%u verschiodenen Zolten mit suften einor gnnzon Railio von Trockonhofoprilpiralon ungestellt, dio ich nach dor Vorsclurift Lob edows aus Berliner Reinzuchtuntorhofo (Hochschulbraueroi) gowunn. Die Priipurato waren niemals so gitrwirksam und hallbar, wie die im Jahr 1916 von Schrodor (München) bezogenen.

Melhodik: Zur Messung der gebildoton Kollonsiiture diente wie bisher das Verfahren von Warburg-Dorner:4) Messung des Kohlensäuredrucks in Burcroftschen Blulgasmanometorn nach kräftigem Schütteln. Die Gelitbe können itn 'Thermostaten solbst geschüttelt. worden, daher ist das $\Lambda \mathrm{b}$ wirten des T'emperaturausgleichs nach dem Schülteln nicht

i) Biochon, Zoitachr., Bil. 86, \$. 825 (1918).

2) Pflitgors Archiv Ba. 144, S. 406 (1912).

") Dioso Zailscbr. Bd. 78, S. 147 (1911),

4) Dioso Zcitsclır. Bd. 81, S. 99 (1912). 
erforderlich und es kann mit Leichtigkeit alle 5 Minuten eine Ablesung vorgenommen werden, wenn nötig auch in noch kürzeren Zwischenräumen. Die Berechnung der Kohlensäure geschieht nach der Formel: $\mathrm{cmm} \mathrm{CO}\left(0^{\circ}, 760\right)=\frac{\mathrm{pv}}{10000(1+a \mathrm{t})}$ $+\frac{a p F}{10000}$, wo $p$ die Druckzunahme in mm Manometerflüssigkeit $(10000=1$ Atm.) bedeutet, $v$ Volumen des Gasraums der Gärungsgefäße (in $\mathrm{cmm}$ ), $\mathrm{F}$ angewandte Flüssigkeitsmenge (in cmm), a Absorptionskoeffizient der Kohlensäure in der Flüssigkeit bei der Versuchstemperatur ( $t$ ). Diese war $25^{\circ}$. $\mathrm{Da}$ das Volumen der Gefäße stets $43 \mathrm{ccm}$ betrug, so ergibt die abgelesene Zahl Millimeter mal 4 ziemlich genau die gebildeten $\mathrm{cmm}$ Kohlensäure $\left(0^{\circ}, 760\right)$.

Um die Kohlensäurebildung vom ersten Moment der $\mathrm{Zu}-$ gabe von Zucker und Phosphat zum Saft verfolgen zu können, wurden die üblichen zylindrischen Druckgefäße mit sackförmigen Anhängen versehen und in diese Anhänge Zucker-, Phosphatlösung und etwaige weitere Zusätze getan, auf den Boden der Gefäße dagegen der Hefesaft. Erst nach Schluß des Manometerhahns wird der Inhalt des Anhangs in den Saft umgekippt, und da dieser keine Selbstgärung aufweist, so wird die ganze vom Moment der Vermischung an gebildete Kohlensäure genau bestimmbar. Voraussetzung dieses Verfahrens ist natürlich, daß keinerlei Kohlensäureretention in der Flüssigkeit stattfindet. Um dies zu verhindern, bezw. um in rergleichenden Messungen diese Möglichkeit zu prüfen, wurden die benutzten Lösungen durch Einleiten von Kohlensäure zunächst gesättigt und nach Entfernung des Überschusses durch kräftiges Schütteln in die Gärungsgefäße gefüllt. Es ergab sich jedoch hierbei, daß die Sättigung des Macerationssafts unnötig ist, da er schon sowieso Spuren $\mathrm{CO}_{2}$ enthält und keins retiniert, auch die von den andern minimalen Flüssigkeitsmengen aufgenommene Kohlensäure kann ganz vernachlässigt werden, außer von der Lösung des neutralen Phosphatgemisches (2 Teile $\mathrm{K}_{2} \mathrm{HPO}_{4}+1$ Teil $\mathrm{KH}_{2} \mathrm{PO}_{4}$ ), die daher zuvor mit Kohlensäure auf die angegebene Weise vorbehandelt wurde. Die Lösung des einbasischen Phosphats, das in manchen Versuchen ver- 
wandt wurde, hält keine Kohlensäure zurück. Falls die Gärung nicht vom ersten Moment an genau beobachtet werden sollte, wurde das Gemisch außerhalb des Thermostaten hergestellt und die Messung nach Einhängen der Gefäße ins Wasserbad und Temperaturausgleich begonnen. Die Zahl für die Anfangszeit (bis zum ersten Schütteln) hat bei genaueren Versuchen dann nur einen orientierenden Wert. Die absolute Genauigkeit ist bei kleinen Ausschlägen am größten, die relative dürfte daher ziemlich unabhängig von deren Größe sein. Bei Messungen in sehr kurzen Zeiträumen - 5 Minuten ist die Genauigkeit der einzelnen Ablesung nicht so sehr groß - etwa auf 5-10\% -, erheblich genauer ist das Gesamtresultat, da sich die Ausschläge der einzelnen Intervalle addieren (bis zur Länge der Skala von $300 \mathrm{~mm}$; dann muß wieder auf Null eingestellt werden). Bei dieser Methode, ebenso übrigens bei den meisten andern zur Bestimmung der Gärungskohlensäure, wird der. Umstand vernachlässigt, daß der Macerationssaft und andere Präparate getöteter Hefe nicht nur gären, sondern auch atmen, d. h. Sauerstoff verbrauchen. ${ }^{1}$ ) Diese Vernachlässigung kann geschehen, weil der Sauerstoffkonsum von einer viel kleineren Größenordnung ist, er beträgt in $0,6 \mathrm{ccm}$ genuinem Macerationssaft, wie er für die Versuche benutzt wurde, nur etwa $1 \mathrm{cmm}$ pro 5 Minuten, während die entsprechenden Ausschläge für Kohlensäure, wie wir im folgenden sehen, etwa $50-500 \mathrm{cmm}$ in 5 Minuten ausmachen.

$\mathrm{Zu}$ den Versuchen diente Lävulose puriss. crist. (Merck). Das hexosephosphorsaure $\mathrm{Na}$ wurde durch Umsatz des $\mathrm{Ca}$ Salzes (Bayers "Candiolin») mit Natriumoxalat gewonnen.

\section{I. Über die «Induktion».}

Nach Harden und Young gelten die folgenden Gärungsgleichungen:

1. $2 \mathrm{C}_{6} \mathrm{H}_{12} \mathrm{O}_{6}+2 \mathrm{PO}_{4} \mathrm{HR}_{2}=2 \mathrm{CO}_{2}+2 \mathrm{C}_{2} \mathrm{H}_{5} \mathrm{OH}+2 \mathrm{H}_{2} \mathrm{O}$ $+\mathrm{C}_{6} \mathrm{H}_{10} \mathrm{O}_{4}\left(\mathrm{PO}_{4} \mathrm{R}_{2}\right)_{2}$.

2. $\mathrm{C}_{6} \mathrm{H}_{10} \mathrm{O}_{4}\left(\mathrm{PO}_{4} \mathrm{R}_{2}\right)_{2}+2 \mathrm{H}_{2} \mathrm{O}=\mathrm{C}_{6} \mathrm{H}_{12} \mathrm{O}_{6}+2 \mathrm{PO}_{4} \mathrm{HR}_{2}$.

1) Pflügers Archiv, Bd. 170 (1918). 
Die Richtigkeit dieser Gleichungen ist von den Autoren durch eine Fülle von Tatsachen gestützt und kann als sichergestellt $\left.{ }^{1}\right)$ gelten. Überdies ist sie auch in einer Nachprüfung von Euler und Johansson ${ }^{2}$ ) bestätigt worden. Natürlich zerfällt der Zucker nach Gleichung I über Zwischenstufen und es kann durch sie nicht behauptet sein, daß dieser Zerfoll unter allen Umständen bis zu den Endprodukten erfolge und daher die Bildung des Hexosephosphats von der Kohlensäurebildung unabtrennbar sei, noch auch, daß die zeitliche Verknüpfung beider Prozesse zu einem durchgängigen vollkommenen Synchronismus führe, da es gut möglich erscheint, daß die Phosphatveresterung nur mit den ersten Phasen der Umwandlung des zweiten Zuckermoleküls verkoppelt ist, die etwaige Verzögerung oder Hemmung der Kohlensäurebildung aber auf einem späteren Stadium des Zuckerzerfalls stattfindet.

Nach den Versuchen der englischen Forscher ist ferner im Hefepreßsaft von den oben formulierten Reaktionen die der Gleichung II während der Hauptzeit des Versuchs die langsamst verlaufende, und da gewöhnlich durch die Selbstgärung des Safts das freie Phosphat zu Beginn schon fast vollständig verestert ist, die Gärungsgeschwindigkeit während der üblichen mehrstündigen oder gar mehrtägigen Versuche bei einem Überschuß an Zucker ganz und gar durch die Zerfallsgeschwindigkeit des Esters, also die Hexosephosphatase bedingt, bis auf das Ende des Versuchs, wo die in Reaktion I wirksamen Zymase und Coferment allmählich zerstört werden, während die Hexosephosphatase sie überlebt. Bei den nach der Buchnerschen Methode angestellten Versuchen beherrscht also die Esterhydrolyse das Bild - von extremen Fällen abgesehen; nur zu Anfang und gegen Ende des Versuchs, bei dem allmählichen Verschwinden von Coferment und Zymase treten andere Faktoren mit ins Spiel. Die Kinetik der eigentlichen Zymasereaktion (I) können wir nur kennen lernen, wenn wir in den ersten Versuchsstunden, wo Zymase und Coferment

1) Zusammenfassung: Alcoholic Fermentation, S. $38 \mathrm{ff}$. Ferner Biochem. Zeitschr. Bd. 40, S. 458 (1912).

2) Diese Zeitschr. Bd. 85, S. 192 (1913). 
noch intakt sind, durch Überschub ron Phosphat und Zucker dafür sorgen, daß die Gärungsgeschwindigkeit ron Gleichung II unabhängig wird.

Nun besitzt aber der Mracerationssaft in Unterschied rom Preßsaft infolge der mangelnden Selbstgürung einen betriichtlichen Vorrat an freiem durch Nagnesiamixtur fällbaren Phosphat - vermehrt vielleicht noch durch Spaltung organischer Phosphorsäureverbindungen während der Trocknung und Macerierung der Hefe -, so fanden Harden und Young in $20 \mathrm{ccm}$ Saft aus Münchener Hefe $\left.0,313 \mathrm{~g} \mathrm{Mg}_{9} \mathrm{P}_{9} \mathrm{O}_{7},{ }^{1}\right)$ ich selbst in der gleichen Menge Saft aus Berliner Hefe $0,258 \mathrm{~g}$; also etwa 0,12-0,14 m Phosphorsäure pro Liter, Zahlen, die natïrlich je nach geringen Modifikationen der Herstellung des Safts schwanken, der Größenordnung nach aber immer gleich sind. Es findet daher auch ohne äußeren Phosphatzusatz zunüchst eine starke Beschleunigung der Gärung statt, bis alles freie Phosphat gebunden ist. Diejenigen Veränderungen dieser «Phosphatperiode» der Gürung, die sich bei weiterem Phosphatzusatz und unter anderen Umständen ergeben, werden im nächsten Kapitel untersucht werden.

Ehe aber diese beschleunigte Angärung vonstatlen geht, kann unter bestimmten leicht erzielbaren Versuchsbedingungen ein kürzeres oder längeres Intervall liegen, in dem keine Kohlensäure gebildet wird und keine Drehungsünderung des Zuckers nachweisbar ist. Dies Intervall, das sich aus einem einfachen Grunde nicht beim Preßsaft, sondern nur beim Macerationssaft, schon weniger bei der zu seiner Herstellung dienenden Trockenhefe finden läßt, wollen wir mit Lebedew, der es am Macerationssaft zuerst beobachtet hat, die In duktion nennen. ${ }^{9}$ ) Der Grund, weshalb man diese nur am Macerationssaft finden kann, ist der, daß sie durch eine Spur Hexosephosphat momentan aufgehoben wird. Hierfür genügt die Konzentration von 1/5 Millimol im Gärungsgemisch, während die Hälfte nicht mehr sicher wirksam ist. Sobald aber ein Gemisch Selbstgärung aufweist, enthält es von vorneherein

1) Biochem. Zeitschr. Bd. \$0, S. 458 (1912).

2) Ann. de l'Institut Pasteur Bd. 26, S. 16 fr. (1912). 
Hexosephosphorsäure und kann daher die Induktion nicht zeigen. Anderseits ist aber die Abwesenheit des Esters zwar eine notwendige, aber noch keine hinreichende Bedingung für die Gärverzögerung. Sie wird vielmehr bei gärkräftigem Saft nur unter bestimmten Umständen beobachtet. Allgemein erleichtern alle Faktoren das Zustandekommen der Induktion, die den im folgenden Kapitel untersuchten sGäranstieg > verlangsamen und vice versa; die Induktionszeit ist um so länger, je stärker dieser Einfluß ist, bis die Angärung schließlich überhaupt ausbleiben kann. Diese Faktoren sind: Verdünnung des Saftes, Erhöhung der Phosphatkonzentration, in geringerem Grad Erhöhung der Zuckerkonzentration; ferner alle Einwirkungen, die die Zymase schädigen, wie längeres Stehenlassen des Safts ohne Zucker usw. Von erheblichem Einfluß ist die Art des Zuckers: Bei gleichen Konzentrationen ist die Induktionszeit bei Saccharose bedeutend kürzer als bei Glukose oder Fruktose, die unter sich ziemlich gleich sind. Manchmal wird auch die Induktion abgekürzt durch ein Gemisch der beiden Hexosen gegenüber jeder einzelnen von gleicher Konzentration; dieser Effekt ist aber weder so stark, noch so regelmäßig, wie das Verhalten des Rohrzuckers.

Die Induktionszeit unabhängig von den genannten Momenten abzukürzen oder aufzuheben, wurde auf zweierlei Weise versucht, durch Beeinflussung des Zuckers und durch Beeinflussung der Hefe. Gehen wir von dem Fall aus, wo die Induktionszeit am längsten ist: mäßig gärwirksamer, verdünnter Saft mit hohem Phosphat- und Fruktosegehalt, so zeigt sich keine Veränderung, wenn der Zucker für sich oder mit Alkali aufgekocht oder mehrere Stunden im Wasserbad auf 70-90 erwärmt wurde; ebensowenig, wenn der Zucker mit Phosphat kurze Zeit gekocht war. Wurde dagegen die Fruktose und ebenso Glukose zusammen mit Phosphat (10 ccm 20\% ige Fruktose $+10 \mathrm{ccm} \mathrm{m} / 2$-neutrales Phosphatgemisch) 4-6 Stunden oder länger bei etwa $80^{\circ}$ gehalten, so war jetzt die Induktion bedeutend verkürzt. Noch günstiger schien es zu sein, wenn ein solches Zuckerphosphatgemisch nach der Erwärmung einige Tage unter Toluol auf Eis gehalten wurde. 
Niemals aber wurde die Induktion so prompt aufgehoben, wie durch Hexosephosphat über $1 / 5$ Millimol, sondern Fruktose und Glukose glichen in dieser Hinsicht nun etwa dem Rohrzucker. Sollte also die Abkürzung der Induktionszeit auf der Bildung von etwas Hexosephosphat beruhen, so müßte es sich unterhalb der genannten Konzentration halten.

Anderseits konnte auch durch eine bestimmte Behandlung der Hefe die Induktionszeit sehr verkürzt oder aufgehoben werden, ohne im übrigen die Gärwirksamkeit des Saftes zu verbessern, nämlich durch trockenes Zerreiben mit Glaspulver vor Herstellung des Safts. Auf diese Weise wird offenbar ein für die Angärung erforderlicher Stoff (vielleicht auch Hexosephosphat) leichter aus den Zellen extrahierbar, der sonst in ihnen zurückbleiben kann. Das wird auch dadurch wahrscheinlich, daß die Trockenhefesuspension selbst unter gleichen Konzentrationsbedingungen keine oder nur eine ganz kurze Induktion aufweist. Bezüglich der im folgenden gegebenen Beispiele sei bemerkt, daß die verschiedenen Versuche, die sogar mit verschiedenen Hefepräparationen angestellt sind, untereinander nicht vergleichbar sind, da die Induktion von geringen Modifikationen des Gärgemisches sehr abhängig ist. Innerhalb der einzelnen Versuchsserie muß daher Vorbehandlung des Safts usw. genau gleich sein. Als Induktion wird die Zeit vom Zusammenmischen der Flüssigkeit bis zur deutlich ansteigenden Kohlensäurebildung angenommen. $\mathrm{CO}_{2}$-Retention findet, wie schon gesagt, nicht statt.

Tabelle I.

\begin{tabular}{|c|c|c|}
\hline $\begin{array}{c}\text { Gemeinsame } \\
\text { Zusammensetzung der } \\
\text { Gemische }\end{array}$ & Variationen & Induktionszeit \\
\hline $\begin{array}{l}\text { 1. Je } 1 \mathrm{ccm}: 0,6 \mathrm{ccm} \text { Saft } \\
+0,1 \mathrm{~m} / 2-\mathrm{KH}_{2} \mathrm{PO}_{4}+0,1 \\
\text { dest. Wasser }+0,2 \mathrm{ccm} \\
\text { Zucker }(20 \%)\end{array}$ & $\begin{array}{c}\text { Fruktose } \\
\text { Saccharose }\end{array}$ & $\begin{array}{c}1^{\mathrm{h}} 45^{\prime} \\
0^{\prime}\end{array}$ \\
\hline $\begin{array}{l}\text { 2. Je } 1,0 \mathrm{ccm}: 0,5 \mathrm{ccm} \text { Saft } \\
+0,35 \mathrm{ccm} \text { dest. Wasser } \\
+\quad \text { Zucker (ohne Phos- } \\
\text { phatzusatz) }\end{array}$ & $\begin{array}{lr}0,15 \text { Fruktose } & 20 \% \\
0,2 & 2 \% \\
0,15 \text { Glukose } & 20 \% \\
0,2 & 2 \% \\
0,15 \text { Saccharose } & 20 \%\end{array}$ & $\begin{array}{l}40^{\prime} \\
15^{\prime} \\
40^{\prime} \\
30^{\prime} \\
0^{\prime}\end{array}$ \\
\hline
\end{tabular}


Tabelle I (Fortsetzung).

\begin{tabular}{|c|c|c|}
\hline $\begin{array}{c}\text { Gemeinsame } \\
\text { Zusammensetzung der } \\
\text { Gomische }\end{array}$ & Variationen & Induktionszeit \\
\hline $\begin{array}{l}\text { 3. Je } 0,9 \mathrm{ccm}: 0,4 \mathrm{ccm} \text { Saft } \\
+0,15 \mathrm{ccm} 20 \% \text { Zucker } \\
+ \text { Wasser }\end{array}$ & $\begin{array}{c}0,15 \text { Fruktose } 20 \% \text { ohne } \\
\text { Phosphat } \\
0,15 \text { Fruktose } 20 \%+0,15 \\
\text { m/2 neutr. Phosphat } \\
\text { do. zusammen gekocht } \\
\text { do. } 6 \mathrm{~h} \text { Wasserbad } 70-80^{\circ} \\
\text { do. } 6 \mathrm{~h} \text { Wasserbad } 70-80^{\circ} \\
\text { (andere Herstellung) } \\
0,15 \text { Glukose } 20 \%+0,15 \\
\text { m/2-n-Phosphat } \\
\text { do. } 6 \mathrm{~h} \text { Wasserbad } 70-80^{\circ}\end{array}$ & $\begin{array}{c}0^{\prime} \\
2-3 \text { Stunden } \\
\text { in } 3 \text { Stund. nicht } \\
\text { angegoren } \\
50^{\prime} \\
40^{\prime} \\
\text { 2-3 Stunden } \\
60^{\prime}\end{array}$ \\
\hline $\begin{array}{l}\text { 4. Je } 1,0 \mathrm{ccm}: 0,5 \mathrm{ccm} \text { Saft } \\
+0,15 \text { Fruktose } 20 \% \\
+0,15 \mathrm{~m} / 2 \text { neutr. Phos- } \\
\text { phat }\end{array}$ & \begin{tabular}{|l} 
Fruktose + Phosphat \\
sammen gekocht \\
do. $2^{\text {h }}$ Wasserbad $70-80^{\circ}$ \\
\end{tabular} & \begin{tabular}{|c} 
in $4^{1 / 2}$ Stunden \\
nicht angegoren \\
do. \\
2 h \\
\end{tabular} \\
\hline $\begin{array}{l}\text { 5. Je } 1,0 \mathrm{ccm}: 0,5 \mathrm{ccm} \text { Saft } \\
+0,15 \mathrm{~m} / \mathrm{s} \text { neutr. Phos- } \\
\text { phat }+0,15 \text { Zucker } 20 \%\end{array}$ & 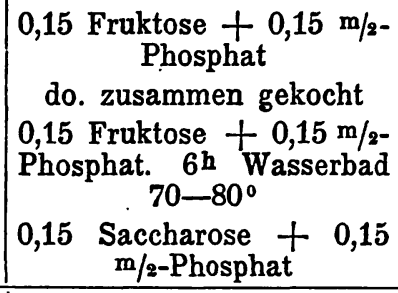 & 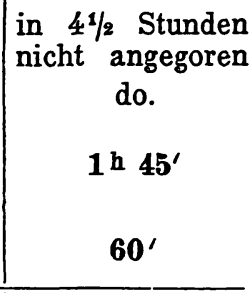 \\
\hline $\begin{array}{l}\text { 6. Je } 0,9 \mathrm{ccm}: 0,6 \mathrm{ccm} \text { Saft } \\
\text { (aus } 1 \text { Jahr alter Münchn. } \\
\text { Hefe) + } 0,15 \text { m } / 2 \text {-Phos- } \\
\text { phat }+0,15 \text { Zucker } 20 \%\end{array}$ & $\begin{array}{c}\text { 0,15 Fruktose }+0,15 \mathrm{~m} / \mathrm{z}- \\
\text { Phosphat } \\
\text { do. } 6 \mathrm{~h} \text { Wasserbad, } 5 \text { Tage } \\
\text { auf Eis (Toluol) } \\
\text { do. (ohne Toluol) } \\
\text { 0,15 Saccharose + 0,15-m/2- } \\
\text { Phosphat }\end{array}$ & $\begin{array}{c}\text { In } 3^{\mathbf{h}} 40^{\prime} \text { nicht } \\
\text { angegoren } \\
40^{\prime} \\
40^{\prime} \\
30^{\prime}\end{array}$ \\
\hline $\begin{array}{l}\text { 7. Je } 1,0 \mathrm{ccm}: 0,6 \mathrm{ccm} \text { Saft } \\
0,1-\mathrm{m} / 2-\mathrm{KH}_{2} \mathrm{PO}+0,1 \\
\text { dest. Wasser }+0,2 \text { Fruk- } \\
\text { tose } 20 \%\end{array}$ & $\begin{array}{l}\text { Hefe unzerrieben } \\
\text {, zerrieben }\end{array}$ & $\begin{array}{c}1 \mathrm{~h} 45^{\prime} \\
10^{\prime}\end{array}$ \\
\hline $\begin{array}{l}\text { 3. Je } 1,0 \mathrm{ccm}: 0,6 \mathrm{ccm} \\
\text { (schlecht wirksamer } \\
+0,25 \text { dest. Wass. } \\
\text { Fruktose } 20 \%\end{array}$ & $\begin{array}{l}\text { Hefe unzerrieben } \\
\text {, zerrieben }\end{array}$ & $\begin{array}{l}\text { In } 3^{1 / 2} \mathbf{h} \text { nicht an- } \\
\text { gegoren } \\
11 / 2-2 \text { Stunden }\end{array}$ \\
\hline
\end{tabular}


Das Phänomen der Induktion ișt nicht identisch, hat aber eine gewisse Verwandtschaft mit der von Euler und Mitarbeitern studierten Angärungshemmung, ${ }^{1}$ ) die sich im Extrakt bestimmter Trockenhefen findet, welcher nicht zur Kohlensäurebildung, sondern nur zur Veresterung des Zuckers mit dem Phosphat befähigt ist. Diese Veresterung kann nämlich auch nicht unmittelbar vonstatten gehen, sondern nur, wenn der Zucker kurze Zeit zuror mit lebender Hefe zusammengehalten, «angegoren» wurde. Euler postuliert ein besonderes Ferment "Hexase», das für die Umwandlung des Zuckers in veresterbare Form erforderlich sei. Nach neuerdings veröffentlichten Versuchen soll aber diese «Angärung» nur für Glukose und Mannose, nicht für Fruktose von nöten sein, ${ }^{2}$ ) es scheint sich also dabei um eine Verwandlung in Fruktose zu handeln. Eine solche der Veresterung vorhergehende Umwandlung des Zuckers in Fruktose kommt in meinen Versuchen natürlich nicht in Betracht, da sich Fruktose und Glukose fast gleich verhalten. Auch kann es sich in dem von mir untersuchten Fall der Gärverzögerung nicht um Bildung des in Alkaligegenwart entstehenden Enols handeln, da dies die Induktionszeit nicht verkürzt; und falls überhaupt während der Induktion eine Veränderung der Hexose stattfindet, könnte zunächst nur sehr wenig Zucker umgewandelt werden, da sich die optische Drehung bis zum Moment der Angärung nicht merklich ändert. Wie wir uns aber insbesonders die Wirkung des Hexosephosphats zu denken haben, soll erst nach Besprechung weiterer Versuche im nächsten Kapitel erörtert werden.

\section{Der * Gäranstieg *}

Die Gärung im Macerationssaft beginnt stets mit einer starken Beschleunigung, bedingt durch das im Saft vorhandene Phosphat. Nach Maßgabe, wie das Phosphat verestert wird,

1) Euler und Ohlsén, Biochem. Żeitschr., Bd. 37, S. 313 (1911). Euler und Kullberg, Diese Zeitschr., Bd. 74, S. 15 (1911). - Euler und Johansson, Diese Zeitschr., Bd. 80, S. 205 (1912). (1917).

2) Euler, Ohlsén, Johansson, Biochem.Zeitschr., Bd. 84, S. 403 
sinkt die Gärungsgeschwindigkeit, bis sie auf einen längere Zeit konstanten Wert fällt, dessen Größe durch die in der Zeiteinheit aus dem Hexosephosphat wieder abgespaltene Phosphatmenge bestimmt wird. Wie viel Kohlensäure unter der Wirkung des freien Phosphats mehr gebildet wird, als in gleichem Zeitraum des späteren Stadiums, ist durch die Hardensche Gleichung I als äquimolekular der Phosphorsäure bestimmt. Aber über die Geschwindigkeit, mit der diese Mehrbildung erfolgt, sagt natürlich die chemische Reaktionsgleichung nichts aus. Der Abfall der Reaktionsgeschwindigkeit zur Zeit, wo die Phosphatkonzentration offenbar im Sinken ist, beweist, daß beide unter gewissen Umständen gleichsinnig verlaufen können. Würden diese Umstände allgemein gelten, so müßte 1. die maximale Gärgeschwindigkeit um so größer sein, je höher man den Gehalt an freiem Phosphat wählt und 2. die Anfangsgeschwindigkeit in der «Phosphatperiode» der Gärung am größten sein, da ja von vornherein die Phosphatkonzentration sinkt. Beides ist nicht der Fall. Schon Harden und Young haben beobachtet, daß 1. die maximale Gärungsgeschwindigkeit bei niederem Phosphatgehalt ziemlich gleich, bei höherem dagegen sehr viel kleiner ist; hoch und niedrig sind relativ, in den verschiedenen Preßsäften wechselnd. In einem typischen Fall ${ }^{1}$ ) verlangsamt sich $z$. B. die maximale Gärgeschwindigkeit (mit 10\% Glukose) in Gegenwart von $0,1 \mathrm{~m}$-Phosphat auf etwa die Hälfte gegenüber $0,067 \mathrm{~m}$-Phosphat, das mit 0,033 m-Phosphat gleich wirkt; 2. sahen sie, daß nach Phosphatzusatz die Geschwindigkeit nicht sofort auf den Maximalwert hinaufschnellt, sondern allmählich ansteigt, am langsamsten bei hoher Phosphatkonzentration. Dabei wird aber das Maximum nicht bei ein und derselben aktuellen Konzentration freien Phosphats erreicht. Der sich hier vom kinetischen Standpunkt ergebenden Schwierigkeiten wissen sie nur dadurch Herr zu werden, daß sie die Annahme machen, der Zucker bilde mit hohen und niederen Phosphatkonzentrationen verschiedene und verschieden stabile Ester, die sich nach Maßgabe des Phosphatverbrauchs in einander umwandelten. Wir werden am Schluß

1) Proc. Roy. Soc. B., Bd. 80, S. 308 (1908). 
dieses Abschnittes sehen, daß sich die bezeichneten Phänomene durch einfachere Annahmen erklären lassen.

Wir betrachten zunächst das anfängliche Steigen der Gärgeschwindigkeit, wir nennen es den *Gäranstieg *, der von den englischen Forschern nicht näher untersucht worden ist. Dieser Gäranstieg hat unmittelbar nichts mit der im vorigen Kapitel beschriebenen Induktion zu tun. Unter allen Umständen, auch bei Gegenwart von Hexosephosphat steigt die Gärungsgeschwindigkeit allmählich zum Maximum. Wiederum wurde auf verschiedene Weise festgestellt, daß dabei keine $\mathrm{CO}_{2}$-Retention eine Rolle spielt, vielmehr beobachtet man genau dasselbe, wenn man einen Überschuß von Kohlensäure einleitet und erst im Apparat fortschüttelt.

Bei sonst ganz gleichen Verhältnissen erfolgt der Gäranstieg um so rascher, je kleiner die Phos$\mathrm{cmm} \mathrm{CO}$

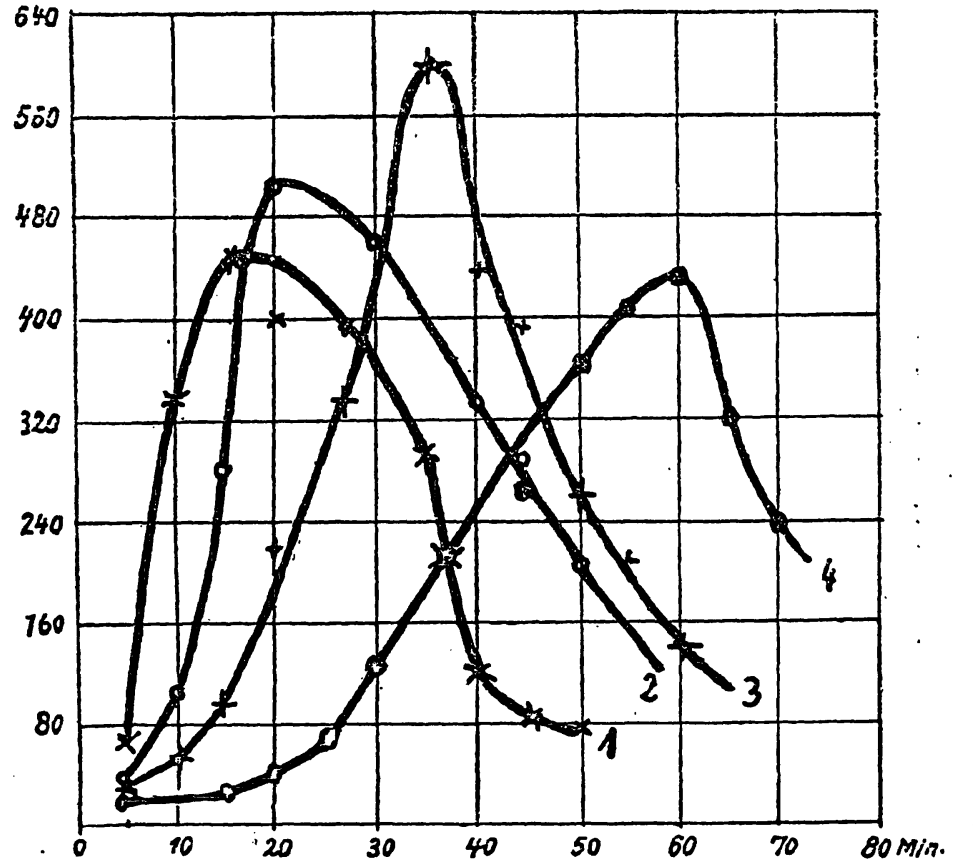

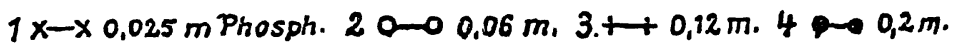

Fig. 1. 
phatkonzentration ist und unabhängig davon, wie groß das Maximum der Gärungsgeschwindigkeit ist.

Dies Resultat sieht man sehr deutlich aus Fig. 1, wo die einzelnen Kurven die Gürungsgeschwindigkeit ( $\mathrm{cmm} \mathrm{CO}_{2}$ pro 5 Minuten) bedeuten bei Zusatz von Phosphat in einer Gesamtkonzentration von (1) $0,025 \mathrm{~m}$, (2) $0,06 \mathrm{~m}$, (3) $0,12 \mathrm{~m}$, (4) 0,2 $\mathrm{m}$ zu einem Gemisch von 1,2 ccm, bestehend aus $0,6 \mathrm{csm}$ Hefesaft, $0,1 \mathrm{ccm}$ Hexosephosphat $(0,05 \mathrm{~m}), 0,2 \mathrm{ccm} 20 \%$ ige Fruktose (Lävulose puriss. crist. Merck), das als Bestandteil des Macerationssafts außerdem schon freies Phosphat entsprechend einer Gesamtkonzentration von $0,06 \mathrm{~m}$ von vorneherein enthält. Die Geschwindigkeit ist vom Moment des Gärungsbeginns an, jeweils für die vorangegangenen $5 \mathrm{Mi}$ nuten aus den Drucksteigerungen in dieser Zeit bestimmt (bei einzelnen ungenaueren Ablesungen ist das Mittel aus zwei benachbarten benutzt). Am steilsten ist der Anstieg, wenn überhaupt kein Phosphat mehr zum Saft zugesetzt wird.

Auf diese Abhängigkeit der Anfangsbeschleunigung der Gärung vom Phosphatgehalt wirft nun die Tatsache ein gewisses Licht, daß jedes andere Salz genau so wirkt wie die Steigerung der Phosphatkonzentration: es verlangsamt den Gäranstieg der Phosphatperiode. Dies zeigt Fig. 2, wo die Gärungsgeschwindigkeiten ( $\mathrm{cmm} \mathrm{CO}_{2}$ pro 10 Minuten) von Gemischen verzeichnet sind, $z u$ denen neben Phosphat $(0,012 \mathrm{~m})$ noch $\mathrm{NaCl}, \mathrm{NaNO}_{3}$ und einbasisches $\mathrm{KH}_{2} \mathrm{PO}_{4}$ hinzugegeben wurde: auf $1,5 \mathrm{ccm}$ mit $0,6 \mathrm{ccm}$ Saft und $0,2 \mathrm{ccm} 20 \%$ iger Fruktose: (1) kein Zusatz, (2) 0,11 m NaCl, (3) 0,2 m NaCl, (4) $0,1 \mathrm{~m} \mathrm{NaNO}{ }_{3}$, (5) $0,1 \mathrm{~m} \mathrm{KH}_{2} \mathrm{PO}_{4}$ (Gesamtkonzentrationen).

Ein zweites Moment führt uns in der gleichen Richtung weiter: Lassen wir den Saft vor Zugabe des Zuckers kurze Zeit mit Phosphat von etwas höherer Konzentration stehen, so erfolgt der Gäranstieg langsamer, aber bis genau zum selben Maximum, wie in einem vollständig gleich zusammengesetzten Gärgemisch, in dem Zucker und Phosphat.zusammen hinzugesetzt wurden. Vergleiche Fig. 3, wo die Gärungsgeschwindigkeít ( $\mathrm{cmm} \mathrm{CO} \mathrm{CO}_{2}$ pro $5 \mathrm{Min}$.) zweier Gemische verglichen ist,

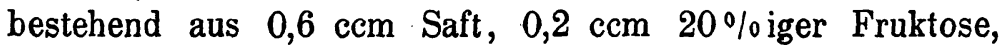


$\mathrm{cmm} \mathrm{CO}$

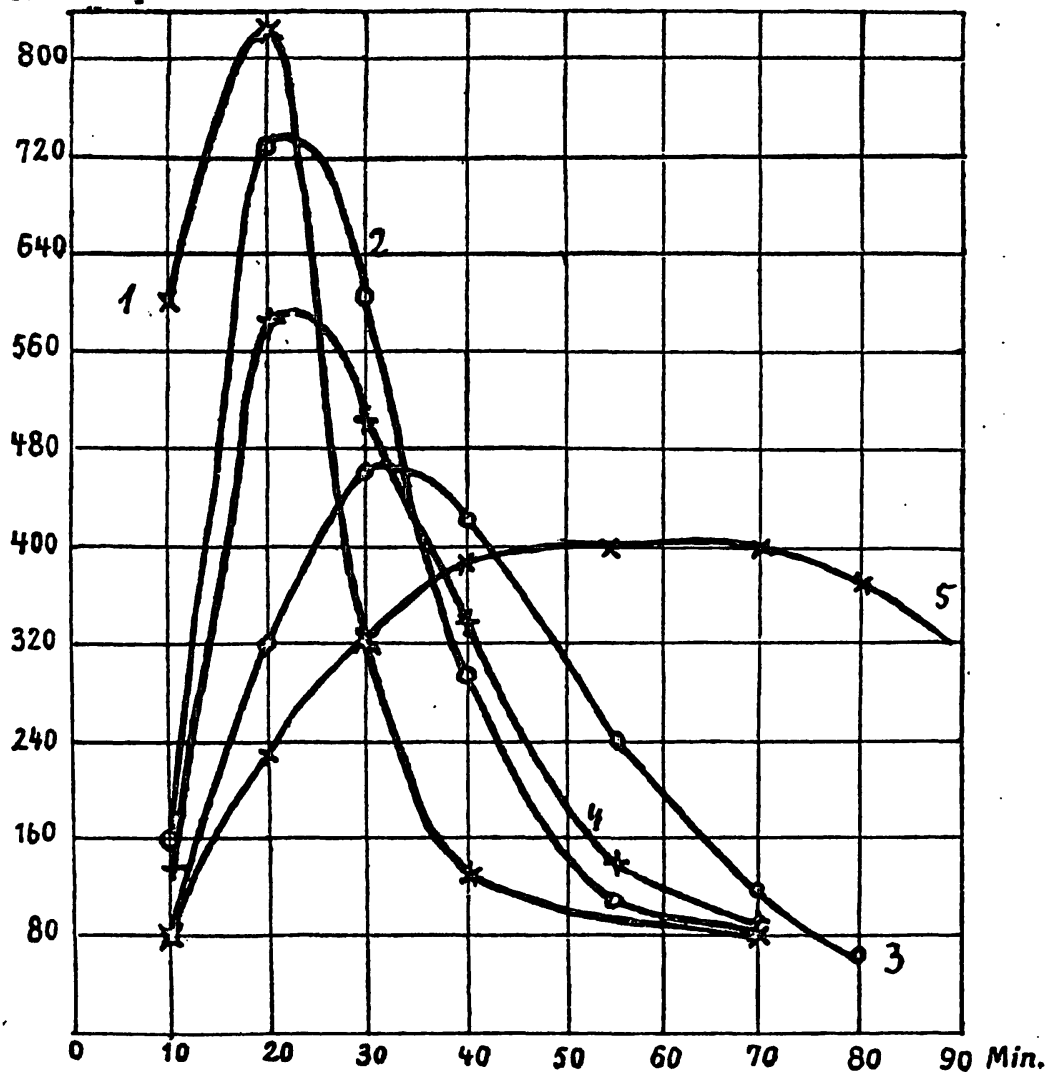

11. $x \rightarrow$ ohne Zusatz $200,11 \mathrm{~m} \mathrm{NaCl} .3 \circ 00,2 \mathrm{~m} \mathrm{NaCl}$.

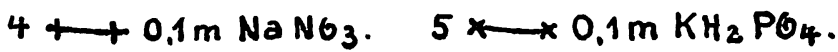

Fig. 2.

$0,2 \mathrm{ccm} \mathrm{m} / 2$ neutr. Phosphat und etwas Hexosephosphat auf $1,4 \mathrm{ccm}$ Flüssigkeit, (1) bei Beginn der Gärung vermischt, während bei (2) das Phosphat 1/2 Stunde vor Beginn zum Saft gegeben wurde.

Aus beiden Versuchsreihen läßt sich der Schluß ziehen: dem Phosphat kommt außer der spezifischen Funktion als Komponente der Gärungsgleichung noch eine allgemeine «Salzfunktion» zu. In dieser Rolle verlangsamt es durch Hemmung oder Schädigung eines Teils des Enzymkomplexes ebenso wie andere Salze irgend eine Phase des Zuckerzerfalls. Für die 


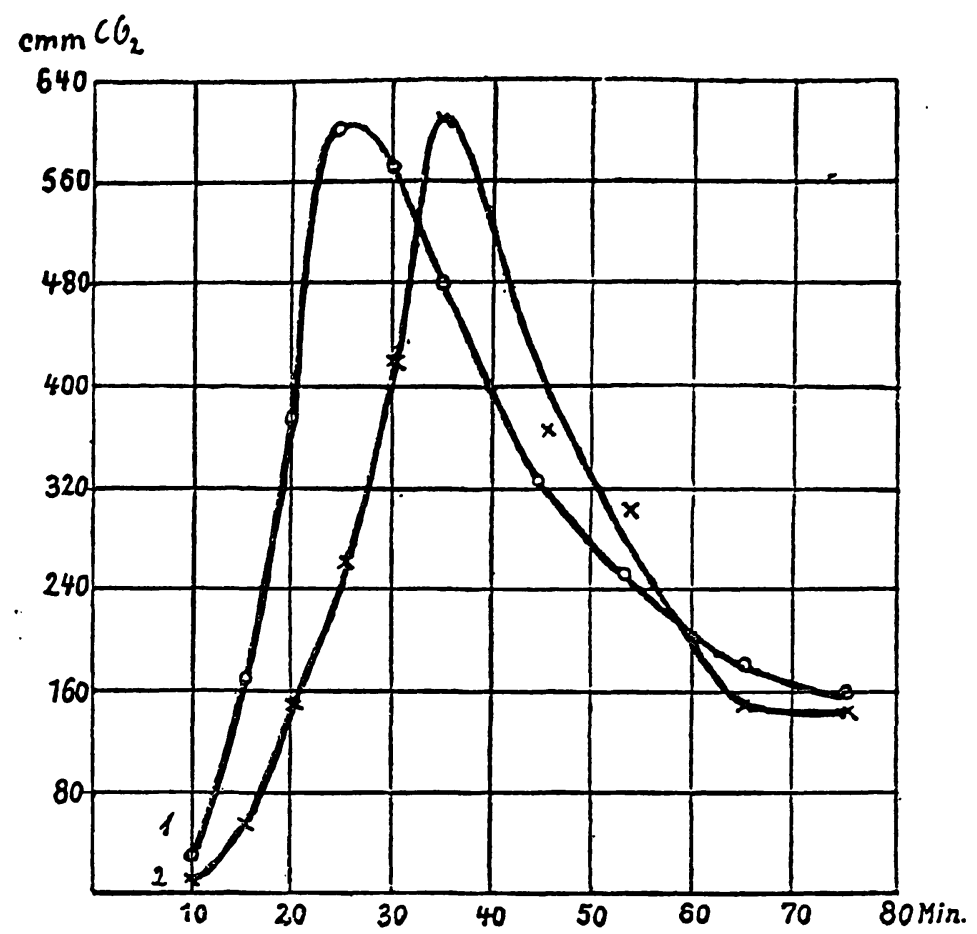

Fig. 3.

verschiedene Form des Gäranstiegs ist also die Annahme der Bildung verschiedener Zuckerphosphorsäuren entbehrlich. Und dasselbe gilt zweifellos ebenso für die Verringerung des $\mathrm{Ge}$ schwindigkeitsmaximums bei hohen Phosphatkonzentrationen. $\mathrm{Daß}$ wir es hier ebenfalls mit einer solchen Hemmung zu tun haben, folgt daraus, daß 1. die Gegenwart eines anderen Salzes das Maximum (bei niedrigem Phosphatgehalt) ebenso herabdrückt wie Vermehrung des Phosphats (vgl. Fig. 2), 2. daß das im ganzen für die Gärung etwas ungünstiger wirkende $\mathrm{KH}_{2} \mathrm{PO}_{4}$ schon bei geringeren Konzentrationen ein abgeflachtes, aber dafür um so länger anhaltendes Gärungsmaximum gibt, wie das neutrale Phosphat. Zwei Unterschiede, die sich zwischen den Gärungskurven mit höherem Phosphatgehalt und mit andern Salzen finden, erklären sich durch die spezifische Phosphatfunktion: durch fremdes Salz wird die Phosphatperiode "nur so viel verlängert, als der Abflachung der Geschwindigkeits- 
kurve entspricht, da ja in beiden Fällen in dieser Periode gleich viel Kohlensäure durch freies Phosphat gebildet wird; geschieht die Abflachung der Kurve durch Vermehrung des Phosphats, so wird die Periode natürlich viel mehr verlängert, weil ja nun viel mehr Kohlensäure in diesem Phosphatstadium der Gärung gebildet wird. Zweitens: Durch anderes Salz wird stets zugleich der Gäranstieg verlangsamt und das Maximum erniedrigt, was bei Vermehrung des Phosphats nicht zu sein braucht. Hier ist in Betracht zu ziehen, daß sich die Phosphatkonzentration ja am Anfang rasch verringert und dadurch Bedingungen für ein weiteres Steigen der Gärgeschwindigkeit gegeben sind, wenn noch freies Phosphat vorhanden ist. (Das gebildete Hexosephosphat hemmt das Maximum auch, aber weniger stark.) Bei sehr geringem Gehalt an Phosphat ist dieses offenbar schon verbraucht, ehe dieses potentielle Maximum erreicht wurde. Dagegen verschwindet das fremde Salz natürlich nicht.

Mit dieser Feststellung ist aber doch noch keine genügende Klarheit über den während des Gäranstiegs vor sich gehenden Prozeß geschaffen. Erinnern wir uns, daß die Angärung durch Hexosephosphat ausgelöst wird. Während des Gäranstiegs nimmt der Hexosephosphatgehalt rasch zu. Sollte hier eine autokatalytische Beschleunigung durch den gebildeten Ester stattfinden? In der Tat: Je mehr Hexosephosphat wir zur Entfesselung der Angärung hinzugeben, um so steiler der Gäranstieg. In ähnlichen Äquivalentkonzentrationen, bei denen anorganisches Phosphat stark verlangsamt, gibt das Hexosephosphat eine Beschleunigung des Gäranstiegs, die besonders deutlich ist bei gleichzeitiger Anwesenheit von viel Kaliumphosphat, weil dann die aufsteigende Kurve ja stark gedehnt ist. Am stärksten sind die Unterschiede bei niederen Konzentrationen des Hexosephosphats. In Fig. 4 ist die im Verlauf des Gäranstiegs gebildete Kohlensäure (Gesamtmenge, nicht Geschwindigkeiten) abgebildet, die entsteht in Gemischen von $1 \mathrm{ccm}$, enthaltend $0,5 \mathrm{ccm}$ Saft, $0,15 \mathrm{~m} / 2$ neutr. Phosphat, 0,15 ccm 20\% ige Fruktose, mit Hexosephosphat in Konzentration von (1) 0,014 m (entspricht 0,028 m-PO ),

Hoppe-Seyler's Zeitschrift f. physiol. Chemie. CII. 


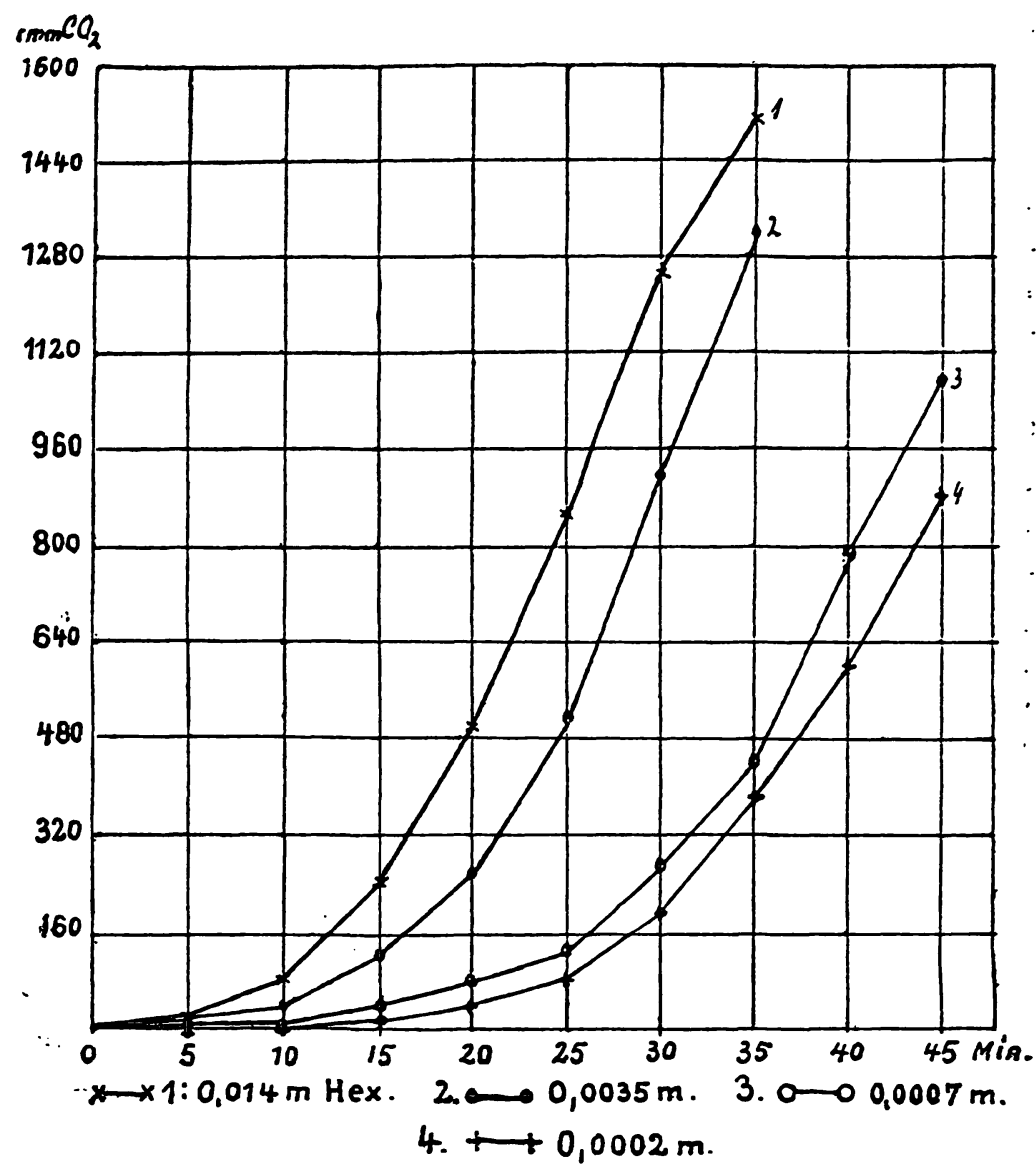

Fig. 4 .

0,0035 m, (3) 0,0007 m, (4) 0,0002 m. Der Gehalt der Stammlösung Hexosephosphat wurde durch Zersetzung mit Salzsäure und Phosphorsäurebestimmung mit Magnesiamixtur ermittelt. Ein Versuch mit ähnlich zusammengesetztem Gärungsgemisch, aber noch höheren Hexosephosphatkonzentrationen ist der folgende. Bei diesem ist bereits das Gärungsmaximum bei der höchsten Konzentration verringert, der Anstieg am Anfang aber gleichwohl beschleunigt:

Zusammensetzung der Gemische : $1,4 \mathrm{ccm}$ Flüssigkeit mit $0,6 \mathrm{ccm}$ Saft, $0,2 \mathrm{ccm} 20 \%$ ige Fruktose, $0,2 \mathrm{ccm} \mathrm{m} / 2$ neutr. Phosphat und Hexose-. phosphat in den angegebenen Konzentrationen. 
Zur Kinetik der zellfreien Gärung.

\begin{tabular}{|c|c|c|c|c|c|c|}
\hline \multicolumn{3}{|c|}{$0,01 \mathrm{~m}$} & \multicolumn{2}{|c|}{$0,02 \mathrm{~m}$} & \multicolumn{2}{|c|}{$0,04 \mathrm{~m}$} \\
\hline & $\begin{array}{c}\text { cmm C } \\
\text { Gesamt }\end{array}$ & $\begin{array}{l}\left.\mathrm{O}_{2} \times 4^{2}\right) \\
\text { pro } 5 \mathrm{Min} .\end{array}$ & $\begin{array}{c}\mathrm{cmm} \\
\text { Gesamt }\end{array}$ & $\begin{array}{l}\mathrm{O}_{2} \times 4 \\
\text { pro } 5 \mathrm{Min} .\end{array}$ & $\begin{array}{c}\text { cmm } \\
\text { Gesamt }\end{array}$ & $\begin{array}{l}\mathrm{O}_{2} \times 4 \\
\text { pro } 5 \mathrm{Min} .\end{array}$ \\
\hline $5^{\prime}$ & 4 & 4 & 7 & 7 & 8 & 8 \\
\hline $10^{\prime}$ & 14 & 10 & 20 & 13 & 24 & 16 \\
\hline $15^{\prime}$ & 32 & 18 & 48 & 28 & 69 & 45 \\
\hline $20^{\prime}$ & 69 & 37 & 126 & 78 & - & 66 \\
\hline $25^{\prime}$ & 119 & 50 & 202 & 76 & 200 & 00 \\
\hline $30^{\prime}$ & 210 & 91 & 294 & 92 & 271 & 71 \\
\hline
\end{tabular}

Daß derselbe Einfluß aber auch bei kleinen Konzentrationen anorganischen Phosphats feststellbar ist, zeigt der folgende Versuch, dessen Geschwindigkeitskurven auf Fig. 5 abgebildet sind.

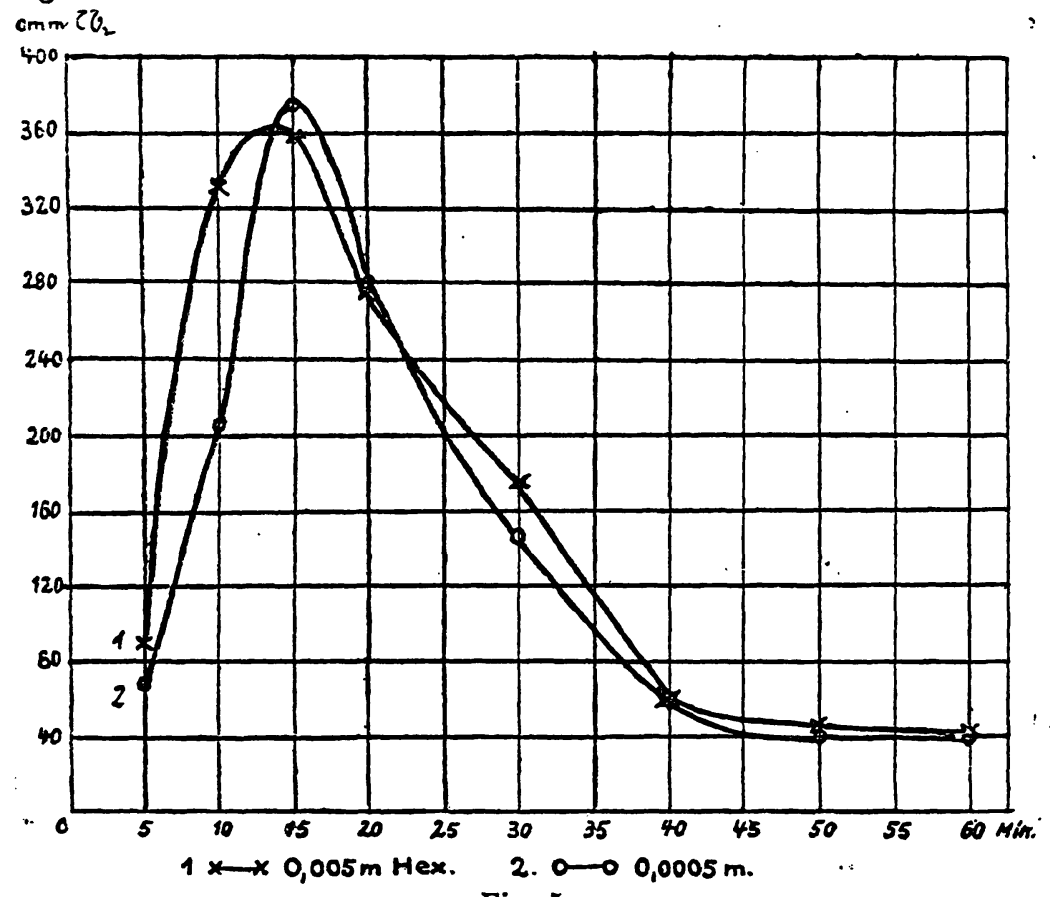

Fig. 5.

Je 1,2 ccm Flüssigkeit, mit 0,5 ccm Saft, 0,2 ccm $20 \%$ iger Fruktose, $0,1 \mathrm{ccm} \mathrm{m} / 5$-Phosphat und Hexosephosphat in der angegebenen Konzentration.

2) Die Zahlen bedeuten: Millimeter-Manometerdruck $=4 \mathrm{cmm} \mathrm{CO}_{2}$. 


\begin{tabular}{|c|c|c|c|c|}
\hline \multicolumn{3}{|c|}{ (1) $0,005 \mathrm{~m}$} & \multicolumn{2}{|c|}{ (2) $0,0005 \mathrm{~m}$} \\
\hline & \multicolumn{2}{|c|}{$\mathrm{cmm} \mathrm{CO}{ }_{2}^{\cdot} \times 4$} & \multicolumn{2}{|c|}{$\mathrm{cmm} \mathrm{CO} \mathrm{CO}_{2} \times 4$} \\
\hline & Gesamt & pro 5 Min. & Gesamt & pro 5 Min. \\
\hline $5^{\prime}$ & 22 & 22 & 17 & 17 \\
\hline $10^{\prime}$ & 105 & 83 & 68 & $\mathbf{5 1}$ \\
\hline $15^{\prime}$ & 194 & 89 & 162 & 94 \\
\hline $20^{\prime}$ & 263 & 69 & 231 & 69 \\
\hline $30^{\prime}$ & 351 & 44 & 302 & 36 \\
\hline $40^{\prime}$ & 381 & 15 & 333 & 15 \\
\hline $50^{\prime}$ & 405 & 12 & 353 & 10 \\
\hline $60^{\prime}$ & 424 & 10 & 372 & 10 \\
\hline
\end{tabular}

Es könnte nach dem Vorangehenden den Anschein haben, als ob sich der Gäranstieg vollständig erklären ließe durch die autokatalytische Wirkung der gebildeten Hexosephosphorsäure in Verbindung mit der «negativ katalytischen * des verschwindenden anorganischen Phosphats. Das ist aber doch nicht der Fall. Denn wie man den Versuch auch einrichtet, niemals setzt die Gärung augenblicklich mit der Maximalgeschwindigkeit ein, sondern steigt - eventuell innerhalb weniger Minuten zum Maximum an. Wählt man die Konzentrationen Ester und freies Phosphat, die sich in einem beliebigen Versuch als im Gärungsmaximum vorhanden errechnen lassen, als Anfangsbedingungen eines zweiten, so beginnt die Gärung dựchaus nicht mit jener Maximalgeschwindigkeit. Ehe wir nochmal auf die Rolle des Hexosephosphats zurückkommen, wollen wir die sich zur Erklärung bietenden Möglichkeiten besprechen. Die einfache Annahme, der Zerfall des Zuckermoleküls erfolge in Etappen, die eine gewisse Zeit beanspruchten bis zur Bildung der Kohlensäure, genügt nicht. Dann müßte nach Verlauf einer gewissen Latenzzeit die Kohlensäurebildung maximal einsetzen. Anders steht es aber mit einem Befunde von Euler und Johansson, ${ }^{1}$ ) der mir in dieser Beziehung wichtig scheint: nach diesen Autoren erfolgt nämlich bei der Gärung von Trockenhefe in Gegenwart von Toluol anfangs die Veresterung des Zuckers rascher als die Bildung der äquivalenten Kohlen-

1) Diese Zeitschr. Bd. 8 s S. 192 (1913). 
säuremenge und erst gegen Schluß der Phosphatperiode findet ein solcher Ausgleich statt, daß die Gesamtmengen äquimolekular werden. Wenn es auch nicht feststeht, wie hoch man die Genauigkeit dieser Versuche ansetzen darf - es ist ja nicht ganz einfach, in Abschnitten von wenigen Minuten unter genau gleichen Bedingungen den Gehalt freien Phosphats und die Kohlensäurebildung jeder Periode zu bestimmen - und die veröffentlichten Versuche der schwedischen Forscher zu einer bestimmten Entscheidung nicht ausreichen, so könnten sie doch in Verbindung mit der hier dargelegten Rolle des Phosphats und Hexosephosphats das Phänomen des Gäranstiegs vom kinetischen Standpunkt verstehen lassen. Die Veresterung würde sofort mit der durch die Konzentration der Komponenten bedingten Geschwindigkeit beginnen, es bestände aber anfänglich eine Dissoziation mit den zur Kohlensäure führenden Zerfallsprozessen des zweiten Zuckermoleküls, die erst allmählich überwunden würde. Dies letztere bleibt natürlich zunächst unaufgeklärt. ${ }^{1}$ ) Daß sich ein - unter gleichen Bedingungen noch steiler verlaufender - Gäranstieg auch bei Trockenhefe findet, zeigt der folgende Versuch.

$4 \mathrm{~g}$ Trockenhefe in $23 \mathrm{ccm}$ Wasser. Davon $1,4 \mathrm{ccm}+0,7$ dest.

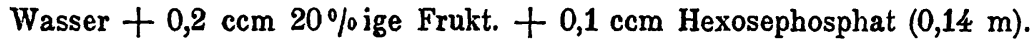
$\mathrm{cmm} \mathrm{CO} \mathrm{CO}_{2} \times 4$

\begin{tabular}{c|c|c}
\hline & Gesamt & pro 21/2 Min. \\
\hline $2^{1} / 2^{\prime}$ & 24 & 24 \\
$5^{\prime}$ & 94 & 70 \\
$7^{1} / 2^{\prime}$ & 269 & 175 \\
$10^{\prime}$ & 314 & 145 \\
$12^{1} / 2^{\prime}$ & 386 & 72 \\
$15^{\prime}$ & 438 & 52 \\
$20^{\prime}$ & 511 & 36 \\
$25^{\prime}$ & 561 & 25 \\
$45^{\prime}$ & 724 & 21 \\
\hline
\end{tabular}

1) Bei ihren Studien über die Phosphatveresterung in dem nicht gleichzeitig gärenden Hefeextrakt haben schon Euler und 0 hlsén, Biochem. Zeitschr., Bd. 37, S. 313 (1911) die Beobachtung gemacht, daß 
Außer der hier gegebenen Deutung des Gäranstiegs muß noch eine andere in Betracht gezogen werden: die Bildung eines Zwischenprodukts vor der endgültigen Veresterung. Dies Zwischenprodukt kann man dann entweder, wie die englischen Forscher, schon als einen intermediären Zuckerphosphorsäureester auffassen, der aber noch nicht mit dem Zerfall des anderen Zuckermoleküls verkoppelt ist, oder als die «veresterbare Form des Zuckers $>$ im Sinn Eulers. Alle meine Versuche in dieser Richtung verliefen negativ. Zucker mit Alkali oder Phosphat gekocht oder längere Zeit auf dem Wasserbad bei $70-90^{\circ}$ erhitzt, auch das die Induktion stark abkürzende Zucker-Phosphatgemisch geben genau den gleichen Gäranstieg, wie nicht vorbehandelter Zucker unter den gleichen Umständen. Auch Inkubation des Zuckers mit nicht gärendem, aber gärfähigem Saft (ohne Hexosephosphat) oder mit Kochsaft hatten kein anderes Resultat. $\mathrm{Daß}$ ein solcher etwaiger intermediärer Körper kein einseitig umgewandelter Zucker, sondern dann schon irgend eine Zucker-Phosphorsäureverbindung sein müßte, geht aus dem Gäranstieg hervor, der bei nachträglichem Phosphatzusatz zum Gärungsgemisch erfolgt. Die Versuche Harden und Youngs sind überhaupt mit Phosphatzusatz zu einem schon in konstanter Gärung befindlichen Preßsaft angestellt: auch dann der allmähliche Anstieg, während doch in diesem Fall schon ein gewisser Überschuß an Zucker vorher in veresterbare Form hätte umgewandelt sein müssen. Beim Macerationssaft geben vergleichende Versuche mit vorherigem und nachträglichem Phosphatzusatz leider kein klares Bild. Einmal werden die Verhältnisse durch die anfängliche Bindung des schon im Saft gegenwärtigen Phosphats stark geändert und dann ist das Gärungsmaximum bei späterem Zusatz immer sehr viel niedriger und daher überhaupt kein einwandfreier Vergleich möglich.

die Geschwindigkeit dieser 'Esterbildung entgegen dem Massenwirkungsgesetz bis zum SchluB nicht abnimmt und daher den szunehmenden Einfluß eines Katalysators oder den <abnehmenden eines Paràlysators> vermutet. Höchstwahrscheinlich ist auch in ihrem Fall das Hexosephosphat dieser Katalysator, das anorganische Phosphat der Paralysator, der Vorgang also eine echte Autokatalyse. 
Die auslösende und beschleunigende Rolle, die das Hexosephosphat bei der Angärung spielt, könnte noch auf eine andere Weise zu erklären versucht werden. Der zugesetzte Ester wird ja vom Hefesaft selbst vergoren, nachdem er zuvor in freies Phosphat und eine fruktoseähnliche Hexose gespalten ist. ${ }^{1)}$ Bei dem großen Unterschied, der sich zwischen Rohrzucker und Fruktose hinsichtlich der Induktion ergab, könnte man annehmen, daß auch die aus dem Hexosephosphat abgespaltene Hexose besonders leicht angegoren werden könnte, etwa weil sie ebenso wie der Invertzucker im Moment der hydrolytischen Spaltung - in statu nascendi - besonders labil wäre, oder auch weil sie sich ohne weitere Umwandlung zur Wiederveresterung mit Phoșphat eignete. Wenn dann auf solche Weise die Gärung in Gang gekommen wäre, würden auch die schwerer angreifbaren Fruktosemoleküle mit in den Zerfall hineingerissen. Manche Angaben in der Literatur über Gärungsaktivatoren sind sogar einer solchen Deutung sehr günstig. So geben M. Oppenheimer ${ }^{2}$ ) und Neuberg ${ }^{3}$ ) an, daß das selbst vergärbare brenztraubensaure Natrium und auch andere ketosaure Salze die Zuckervergärung beschleunigen und wie es nach ihren Angaben scheint, am stärksten zu Beginn der Gärung. Brenztraubensaures Na kann jedoch, obwohl es stürmisch von Macerationssaft vergoren wird, das Hexosephosphat nicht ersetzen. Es läßt sich auch auf einem direkteren Wege zeigen, daß die hier herangezogene, ganz plausible Deutung nicht zutriff. In diesem Fall käme als Beschleuniger des Gäranstiegs die abgespaltene «labile» Hexose in Betracht und der Einfluß der Konzentration des Hexosephosphats auf die Steilheit des Anstiegs müßte auf die Konzentration der Spaltprodukte bezogen werden, die ja - ceteris paribus - mit jener parallel geht. Nun können wir nach einer Entdeckung Harden und Youngs den Gehalt an abgespaltener Hexose stark vermehren bei gleichzeitiger Verringerung der Hexosephosphatmenge, nämlich durch

1) Young, Proc. Roy. Soc. B., Bd. 81, S. 528 (1909).

2) Diese Zeitschr., Bd. 93, S. 235 (1914/15).

s) Biochem. Zeitschr., Bd. 71, S. 1 (1915). 
Natriumarseniat. Die englischen Forscher zeigten, daß die enorme Beschleunigung der zellfreien Gärung durch gewisse Konzentrationen von arsensaurem Natrium auf nichts anderem beruht als starker Steigerung der Hydrolyse des Hexoseesters, durch die der Gehalt an freiem Phosphat dauernd hoch gehalten wird. ${ }^{1}$ ) Dies wurde schon von ihnen auf verschiedene Weise bewiesen. Immerhin verringert die starke Selbstgärung des Preßsafts und die Zunahme, die auch diese infolge vermehrter Hydrolyse des Glykogens durch Arseniat erfährt, die Durchsichtigkeit ihres Beweismaterials. ${ }^{2}$ ) Das Fehlen der Selbstgärung im Macerationssaft gestattet, diese Tatsache in höchst überzeugender Weise zu demonstrieren und gleichzeitig festzustellen, daß die Methode zur Entscheidung der Frage, ob das Hexosephosphat oder seine Spaltprodukte den Gäranstieg beschleunigen, benutzt werden kann.

1. Verwendet man eine niedrige Phosphatkonzentration, die ein hohes Geschwindigkeitsmaximum der Gärung gibt - also eventuell Macerationssaft ohne weiteren Phosphatzusatz -, so erhält man mit und ohne Arseniat dasselbe Maximum; während es aber in Abwesenheit von Arseniat rasch abfällt, bleibt es mit diesem lange bestehen. Ein solcher Versuch ist auf Fig. 6 abgebildet. Hier wurde aus besonderen Gründen, die in diesem Zusammenhang nicht interessieren, statt des Hefesafts sein über dem Ultrafilter gewaschener Rückstand benutzt und Coferment und Phosphat in Gestalt von Macerationskochsaft hinzugegeben. Aus diesem Grund sind die Ausschläge etwas kleiner. Die Kurven bedeuten die Geschwindigkeiten (cmm C. $\mathrm{O}_{2}$ pro 10 Minuten) von Gemischen zu $1,2 \mathrm{ccm}$, enthaltend 0,2 ccm dreifach eingedickten Rückstand, 0,5 Kochsaft, $0,2 \mathrm{ccm} 20 \%$ ige Fruktose, etwas Hexosephosphat. und (1) kein Arseniatzusatz, (2) 0,005 m Natriumarseniat, (3) 0,012 m Natriumarseniat. Man sieht, daß bei dieser höheren Konzentration die Geschwindigkeit etwas langsamer steigt, aber dann im Maximum lange Zeit konstant ist. Vor allem ergibt sich aber sehr deutlich, daß die Arseniatbeschleu-

2) Proc. Roy. Soc. B., Bd. 83, S. 451 (1911).

2) a. a. $0 .$, S. 463. 
nigung erst mit dem Absinken vom Phosphatmaximum einsetzt.

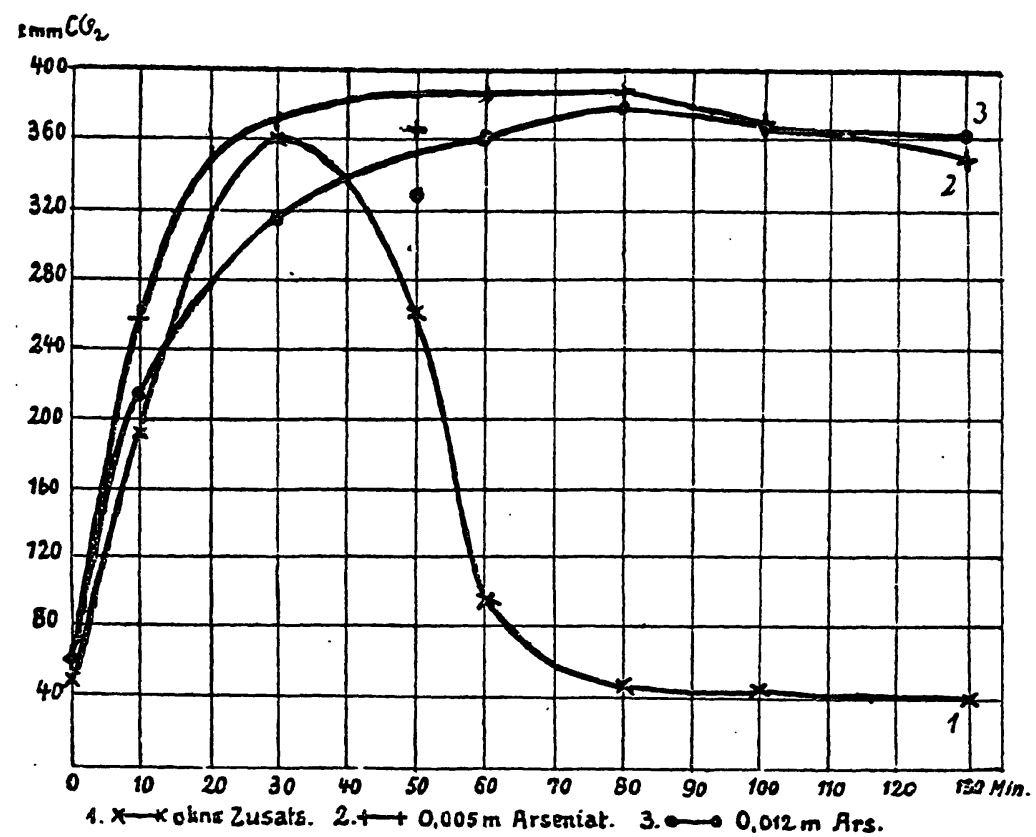

Fig. 6.

2. Läßt man statt Fruktose Hexosephosphat vergären, so ist die Gärgeschwindigkeit bei gleicher Zuckerkonzentration viel geringer und von vorneherein ziemlich konstant, sie zeigt keine aPhosphatperiode»; sie ist bedingt durch die Geschwindigkeit der vorangehenden Hexosephosphatspaltung. Setzt man hierzu nun Arseniat in optimaler Konzentration $(0,01$ bis $0,005 \mathrm{~m}$ ), so wird die Gärungsgeschwindigkeit sofort und ganz außerordentlich stark gesteigert, viel stärker als beim gleichen Hefesaft die Fruktosevergärung nach Ablauf der Phosphatperiode. Bei einem ziemlich schlecht gärenden Saft, bei dem diese letztere nur etwa auf den dreifachen Wert hinaufging, wurde die Hexosephospatvergärung von Anfang an bis zu dem rasch erfolgenden Versiegen des Gärmaterials um den 20 bis 30 fachen Betrag gesteigert; wie die nachstehenden Versuche zeigen.

Versuch 1. Gemische $1,2 \mathrm{ccm}$, enthaltend $0,7 \mathrm{ccm}$ Saft und (1) $0,3 \mathrm{ccm} 0,14 \mathrm{~m}$ Hexosephosphat, (2) $0,3 \mathrm{ccm}$ Hexosephosphat $+0,1 \mathrm{ccm}$ 
$\mathrm{m} / \mathrm{so}$ Arseniat, (3) $0,1 \mathrm{ccm} 20 \%$ Fruktose, (4) $0,1 \mathrm{ccm} 20 \%$ Fruktose $+0,1 \mathrm{ccm} \mathrm{m} / 20$ Arseniat.

$$
\mathrm{CO}_{2} \text {-Bildung : } \mathrm{cmm} \mathrm{CO}_{2} \times 4 .
$$

\begin{tabular}{|c|c|c|c|c|c|c|c|c|}
\hline & \multicolumn{2}{|c|}{ (1) } & \multicolumn{2}{|c|}{ (2) } & \multicolumn{2}{|c|}{ (3) } & \multicolumn{2}{|c|}{ (4) } \\
\hline & $\begin{array}{l}\text { Hed } \\
\text { pho }\end{array}$ & $\begin{array}{l}\text { ose- } \\
\text { phat }\end{array}$ & $\begin{array}{l}\text { Hexo } \\
\text { phat }\end{array}$ & $\begin{array}{l}\text { ephos- } \\
\text { - Ars. }\end{array}$ & Fru & tose & $\begin{array}{c}\text { Fruk } \\
+\end{array}$ & $\begin{array}{l}\text { tose } \\
\text { Ars. }\end{array}$ \\
\hline & $\begin{array}{l}\mathrm{Ge}- \\
\text { samt }\end{array}$ & $\begin{array}{c}\text { pro } \\
10 \mathrm{Min} .\end{array}$ & $\begin{array}{l}\text { Ge- } \\
\text { samt }\end{array}$ & $\begin{array}{c}\text { pro } \\
10 \mathrm{Min}\end{array}$ & $\begin{array}{l}\text { Ge- } \\
\text { samt }\end{array}$ & $\begin{array}{c}\text { pro } \\
10 \mathrm{Min} .\end{array}$ & $\begin{array}{l}\text { Ge- } \\
\text { samt }\end{array}$ & $\begin{array}{c}\text { pro } \\
10 \mathrm{Min} .\end{array}$ \\
\hline $10^{\prime}$ & 4 & 4 & 29 & 29 & - & - & - & - \\
\hline $25^{\prime}$ & 12 & 5 & 210 & 121 & 127 & (51) & 131 & (52) \\
\hline $40^{\prime}$ & 20 & 5 & 280 & 47 & 185 & 39 & 183 & 35 \\
\hline $1 \mathrm{~h}$ & 32 & 6 & 310 & 15 & 317 & 66 & 288 & 53 \\
\hline $1 \mathrm{~h} 15^{\prime}$ & 43 & 7 & 323 & 9 & 375 & 39 & 392 & 69 \\
\hline $1 \mathrm{~h} 35^{\prime}$ & - & - & - & - & 436 & 30 & 541 & 74 \\
\hline $1 \mathrm{~h} 45^{\prime}$ & - & - & - & - & 464 & 28 & 616 & 75 \\
\hline
\end{tabular}

Versuch 2. Gemische 1,2 ccm: enthaltend $0,7 \mathrm{ccm}$ Saft, $0,3 \mathrm{ccm}$ 0,14 m-Hexosephosphat und (1) ohne Arseniat, (2) 0,008 m-Arseniat, (3) 0,002 m Arseniat.

$$
\mathrm{cmm} \mathrm{CO}_{2} \times 4 \text {. }
$$

\begin{tabular}{r|c|c|c|c|c|c}
\hline \hline & $(1)$ & \multicolumn{2}{|c|}{$(2)$} & \multicolumn{2}{c}{$(3)$} \\
\hline & \multicolumn{2}{|c|}{ ohne Ars. } & \multicolumn{2}{c|}{ 0,008 Ars. } & \multicolumn{2}{c}{ 0,002 Ars. } \\
& Gesamt & pro 5 Min. & Gesamt & pro 5 Min. & Gesamt & pro 5 Min. \\
\hline $5^{\prime}$ & 5 & 5 & 21 & 21 & 14 & 14 \\
$10^{\prime}$ & 9 & 4 & 97 & 76 & 71 & 57 \\
$30^{\prime}$ & 20 & $\mathbf{3}$ & 326 & $\mathbf{5 7}$ & 280 & 52 \\
$1 \mathrm{~h}$ & 46 & 4 & 391 & 11 & 370 & 15
\end{tabular}

Versuch 3. Gemische $1,6 \mathrm{ccm}$ enthaltend $0,8 \mathrm{ccm}$ Saft, $0,4 \mathrm{ccm}$ 0,14 m-Hexosephosphat und (1) ohne Arseniat, (2) 0,006 m-Arseniat.

\begin{tabular}{c|c|c|c|c}
\hline & \multicolumn{2}{|c|}{$(1)$} & \multicolumn{2}{c}{$(2)$} \\
\hline \multirow{2}{*}{$\mathrm{CO}_{2} \times 4$} & \multicolumn{2}{|c|}{ ohne Arseniat } & \multicolumn{2}{c}{ mit Arseniat } \\
& Gesamt & pro 10 Min. & Gesamt & pro 10 Min. \\
\hline & 6 & 6 & 122 & 122 \\
$10^{\prime}$ & 15 & 9 & 367 & 243 \\
$20^{\prime}$ & 29 & 7 & 442 & 37 \\
$40^{\prime}$ & 44 & 7 & 472 & 15 \\
$60^{\prime}$ & & & &
\end{tabular}


3. Untersucht man nun den Gäranstieg von Fruktose in Anwesenheit von wenig Hexosephosphat mit und ohne Arseniatzusatz, so wird die Konzentration des Hexosephosphats anfangs durch das Arseniat rasch verringert, während die Konzentration der Spaltprodukte relativ außerordentlich steigt. Diese Vermehrung muß ungefähr so groß sein, wie bei ausschließlicher Hexosephosphatvergärung die Beschleunigung am Anfang beträgt. . Kommt es für die Angärung auf das Hexosephosphat selbst an, so kann das Arseniat keinen beträchtlichen Einfluß haben, und wenn, nur im Sinne einer Verlangsamung, da ja die Hexosephosphatkonzentration sich auch mit Arseniat relativ in den ersten Minuten nicht sehr bedeutend ändert. Käme es aber auf die Spaltprodukte an, so müßte das Arseniat den Gäranstieg außerordentlich beschleunigen, da die relative Menge derselben dadurch sehr wächst. Die genauesten Versuche in Gegenwart von sehr wenig Hexosephosphat so daß dessen Eigengärung keine Komplikation hervorruft - zeigen nun, daß in der Tat kein oder höchstens ein verlangsamender Einfluß des Arseniats auf die Angärung festzustellen ist.

Versuch 4. 1,2 Gemisch, enthaltend $0,6 \mathrm{ccm}$ Saft, $0,2 \mathrm{ccm} 20 \%$ ige Fruktose, 0,0006 m Hexosephosphat, und (1) ohne Zusatz, (2) mit 0,005 Arseniat.

\begin{tabular}{c|c|c|c|c}
\hline \multicolumn{4}{c|}{$\mathrm{cmm} \mathrm{C0} \times 4$} \\
\hline & \multicolumn{2}{|c|}{$(1)$} & \multicolumn{2}{|c}{$(2)$} \\
\hline & \multicolumn{2}{|c|}{ ohne Zusatz } & \multicolumn{2}{|c}{ mit Arseniat } \\
& Gesamt & pro 5 Min. & Gesamt & pro 5 Min. \\
\hline $5^{\prime}$ & 5 & 5 & 4 & 4 \\
$10^{\prime}$ & 21 & 16 & 20 & 16 \\
$15^{\prime}$ & 51 & 30 & 48 & 28 \\
$20^{\prime}$ & 100 & 49 & 94 & 46 \\
$30^{\prime}$ & 211 & 56 & 200 & 53 \\
$40^{\prime}$ & 380 & 84 & 375 & 87 \\
$50^{\prime}$ & 523 & 71 & 511 & 68 \\
$60^{\prime}$ & 596 & 37 & 749 & 69 \\
$70^{\prime}$ & 622 & 13 & 857 & 54 \\
$80^{\prime}$ & 645 & 12 & 953 & 46
\end{tabular}


III. Die Bedeutung des Cofermentgehalts für die Gärgeschwindigkeit.

Welchen Einfluß übt das Coferment auf die Geschwindigkeit aus? Da im Hauptintervall des Gärungsablaufs die Umsatzgröße durch die Hexosephosphatase bedingt wird und diese, wie die englischen Autoren zeigten, des Cofermentes nicht bedarf, so kann sich in diesem Abschnitt eine Vermehrung desselben über die Menge hinaus, die ausreicht, um die Zymase zur völligen Beschlagnahme des abgespaltenen Phosphats za befähigen, gar nicht geltend machen (wenn man nicht gleichzeitig auch die Phosphatmenge vermehrt). Es wird daher hier nur die Wirkung des Cofermentgehalts auf die Gärung in der Phosphatperiode untersucht. Von besonderer Bedeutung erscheint die Frage, ob es für die Geschwindigkeit auf die Menge des Coferments relativ zur Zymase, oder auf seine Konzentration - unabhängig von der Enzymmenge - ankommt, vorausgesetzt, daß überhaupt eine Abhängigkeit vom Cofermentgehalt zu konstatieren ist. Wenn die Menge des Körpers mit der Enzymmenge in einer bestimmten Proportion stehen müßte, so würde das darauf hindeuten, daß er sich chemisch an dem Zuckerumsatz beteiligt, im andern Fall würde seine Bedeutung mehr in der Richtung eines Milieueinflusses zu suchen sein.

Daß innerhalb der hier in Betracht kommenden Verhältnisse zunächst überhaupt eine Abhängigkeit der Geschwindigkeit vom Coenzymgehalt besteht, kann man am deutlichsten feststellen, wenn man nur diesen und nicht gleichzeitig die Phosphatmenge variiert. Dies geschieht am besten durch $\mathrm{Zu}$ satz von Muskelkochsaft, da dieser, wie ich kürzlich gezeigt habe, das Gärungskoferment besitzt, aber eine im Vergleich zum Macerationssaft $\mathrm{zu}$ vernachlässigende Phosphatmenge. ${ }^{1}$ ) Oder man verwendet Hefekochsaft und verringert die zuzugebende Menge Phosphat um den Betrag, der sich für den Kochsaft berechnet. In jedem Fall erfolgt dann der Gäranstieg viel rascher. Ja, dies kann sogar der Fall sein, wenn man ohne weitere Variation Hefekochsaft zum Gär-

1) Diese Zeitschr., Bd. 101, S. 165 (1918) und Bd. 102 (?), S. 1 (1918), Vers: 26. 
gemisch hinzusetzt, obwohl wegen der dadurch gleichzeitig bewirkten Vermehrung des Phosphats das Umgekehrte der Fall sein sollte. Als Beispiel sind in Fig. 7 die Geschwindigkeits-

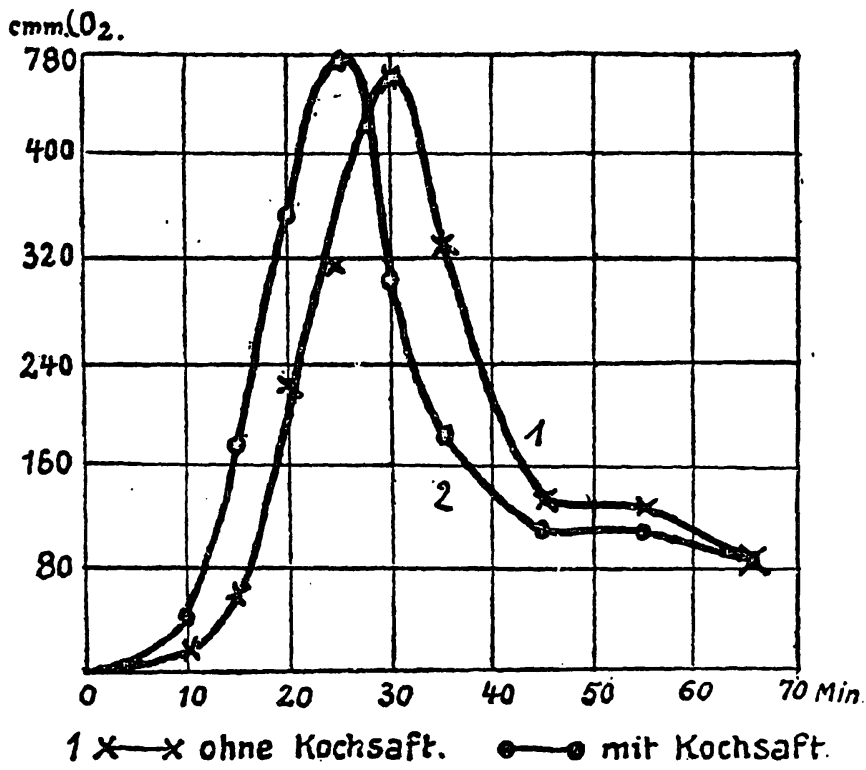

Fig. 7.

kurven ( $\mathrm{cmm} \mathrm{CO}_{2}$ pro 5 Minuten) bei den üblichen Mengenverhältnissen gegeben. $1,4 \mathrm{ccm}$ Gemisch, enthaltend $0,6 \mathrm{ccm}$ Saft, 0,1 ccm 20\% ige Fruktose, etwas Hexosephosphat, zu (1) $0,2 \mathrm{ccm} \mathrm{m} / 2$ Phosphat und dest. W., zu (2) $0,15 \mathrm{ccm} \mathrm{m} / 2$ Phosph. +0,4 ccm Hefekochsaft. Obwohl so die Phosphatkonzentration in (2) noch etwas größer ist als in (1), erfolgt der Gäranstieg steiler. In anderer Weise und unter Bedingungen, die den folgenden Versuchen entsprechen, läßt sich dasselbe nachweisen, wenn man zu gut gewaschenem, phosphat- und cofermentfreiem Ultrafiltrationsrückstand einmal Muskelkochsaft und zweitens im Vakuum eingeengten Muskelkochsaft hinzugibt, außerdem gleich viel Phosphat zu beiden. Wiederum ist der Gäranstieg im zweiten Fall mit konzentrierterem Coferment steiler, obwohl auch hier die Phosphatmenge gleichzeitig etwas vermehrt ist.

Versuch 1. Gemisch $1,2 \mathrm{ccm}$, enthaltend $0,2 \mathrm{ccm}$ mit $400 \mathrm{fach}$ Wasser gewaschenen, dreifach eingedickten Rückstand, 0,2 ccm $20 \%$ ige 
Fruktose, $0,1 \mathrm{ccm} \mathrm{m} / 2-\mathrm{KH}_{2} \mathrm{PO}_{4}$ und etwas Hexosephosphat mit (1) $0,6 \mathrm{ccm}$ Wasser, (2) 0,6 ccm Muskelkochsaft, (3) 0,6 ccm im Vakuum zur Trockne eingedampften, dreifach konzentrierten Muskelkochsaft.

\begin{tabular}{|c|c|c|c|c|c|}
\hline & (1) & & (2) & & (3) \\
\hline & $\begin{array}{c}\text { mit Wasser } \\
\text { Gesamt }\end{array}$ & $\begin{array}{l}\text { mit Mu: } \\
\text { Gesamt }\end{array}$ & $\begin{array}{l}\text { kelkochsaft } \\
\text { |pro15Min. }\end{array}$ & $\begin{array}{r}\text { mit kor } \\
\text { ko } \\
\text { Gesamt }\end{array}$ & $\begin{array}{l}\text { z. Muskel- } \\
\text { chsaft } \\
\text { |pro15Min }\end{array}$ \\
\hline $15^{\prime}$ & 0 & 30 & 30 & 56 & อ̈6 \\
\hline $30^{\prime}$ & 3 & 114 & 84 & 169 & 114 \\
\hline $45^{\prime}$ & 6 & 200 & 86 & 264 & 95 \\
\hline $1 \mathrm{~h} 15^{\prime}$ & 9 & 358 & 79 & 438 & 87 \\
\hline $2 \mathrm{~h} 15^{\prime}$ & 12 & 498 & $3 \tilde{5}$ & 574 & 34 \\
\hline
\end{tabular}

Versuch 2. Höhere Phosphatkonzentration. 1,2 ccm Gemisch, enthaltend 0,2 ccm mit 110 fach Wasser gewaschenen, dreifach eingedickten Rückstand, 0,2 ccm $20 \%$ ige Fruktose, $0,1 \mathrm{ccm} \mathrm{m}-\mathrm{KH}_{2} \mathrm{PO}_{4}$, etwas Hexosephosphat, und (1) 0,6 ccm Wasser, (2) 0,6 ccm Muskelkochsaft, (3) 0,6 ccm im Vakuum doppelt eingedickten Muskelkochsaft.

\begin{tabular}{|c|c|c|c|c|c|}
\hline & (1) & & (2) & & (3) \\
\hline & $\begin{array}{c}\text { mit Wasser } \\
\text { Gesamt }\end{array}$ & $\begin{array}{l}\text { mit Mus } \\
\text { Gesamt }\end{array}$ & $\begin{array}{c}\text { kelkochsaft } \\
\text { |pro15Min. }\end{array}$ & $\begin{array}{r}\text { mit dor } \\
\text { Muske } \\
\text { Gesamt }\end{array}$ & $\begin{array}{l}\text { pelt konz. } \\
\text { lkochsaft } \\
\text { /pro15Min }\end{array}$ \\
\hline $15^{\prime}$ & 4 & 60 & 60 & 108 & 108 \\
\hline $30^{\prime}$ & 11 & 188 & 88 & 280 & 172 \\
\hline $45^{\prime}$ & 24 & 372 & 184 & 495 & 215 \\
\hline
\end{tabular}

Einen Hinweis darauf, daß die Geschwindigkeit von der Konzentration und nicht der Menge des Coferments beeinflußt wird, können wir darin sehen, daß Zusatz von Muskelkochsaft zu stark verdünntem Hefesaft einen viel stärkeren Einfluß auf die Beschleunigung des Gäranstiegs hat, als zu weniger verdünntem, obwohl doch in beiden Fällen das Verhältnis von Zymase und Coenzym dasselbe ist. Immerhin sind solche Versuche nicht einwandfrei, weil sich dabei auch das Verhältnis von Coferment zu Phosphat ändert und zwischen beiden 
offenbar auch Beziehungen bestehen. Um eine sichere Entscheidung zu fällen, muß die Zusammensetzung in bezug auf Zucker, Phosphat und Coferment nebst allen sonstigen Konstituenten des Gärgemisches unverändert bleiben, und nur die Zymasemenge variiert werden. $\mathrm{Zu}$ diesem $\mathrm{Zweck}$ wird gewaschener Ultrafiltrationsrückstand $=$ Zymase in wechselnder Verdünnung zu genau gleichen Gärgemischen hinzugefügt, die aus Muskel- oder Hefekochsaft, Fruktose, Phosphat, etwas Hexosephosphat bestehen. Die Cofermentkonzentration nebst allem übrigen ist dann konstant außer der Zymase, die selbst und daher auch ihr Verhältnis zur Cofermentmenge variiert wird. Wenn die Konzentration des Coferments maßgebend ist, so müssen die Geschwindigkeiten bis zum Abfall des Phosphatmaximums ziemlich genau den Zymasemengen proportional sein, andernfalls müßte bei höherem Zymasegehalt die Geschwindigkeit relativ schwächer ansteigen. $\mathrm{Da}$ die Geschwindigkeit sich ja dauernd ändert, so vergleichen wir nur die gesamte gebildete Kohlensäuremenge bis zu dem Zeitpunkt, wo in der schnellst vergärenden Lösung die Geschwindigkeit zu sinken anfängt. Der Vergleich wird erleichtert bei Benutzung von $\mathrm{KH}_{2} \mathrm{PO}_{4}$, weil hier das Maximum weniger hoch und gedehnter ist. ${ }^{1}$ )

Es ergibt sich, wie die folgenden Versuche zeigen, recht deutlich, daß bis zur Überschreitung des Maximums die Geschwindigkeit den $\mathrm{Zymasemengen} \mathrm{direkt}$ proportional ist, daß mithin die Konzentration und nicht die Coenzymmenge im Verhältnis zur Zymasemenge für dieUmsatzgröße in dieser Zeit maßgebend ist.

1) Es sei hier der Hinweis gestattet, $\mathrm{daB}$ in der letzten Arbeit (diese Zeitschr., Bd. 102, S. 1 [1918]) der Schluß aus der Gärungsgeschwindigkeit der Anfangszeit - cum grano salis - auf den Cofermentgehalt der Organkochsäfte nur erlaubt war, weil erstens durch ziemlich viel $\mathrm{KH}_{2} \mathrm{PO}_{4}$, ein gedehntes, lang anhaltendes Gärungsmaximum erzeugt wurde, und der eigene Phosphatgehalt der Organsäfte relativ wenig in Betracht kam, und zweitens die Zeiten für die Vergleiche im allgemeinen so kurz gewählt wurden, daß die Phosphatsteigerung noch nicht abgelaufen war. In der Zeit des Geschwindigkeitsabfalls überschneiden sich ja sogar bei ursprünglich gleichem Phosphatgehalt die Geschwindigkeitskurven. 
Versuch 1. Je $1,4 \mathrm{ccm}$ Gemisch, enthaltend $0,5 \mathrm{ccm}$ Muskelkochsait, $0,1 \mathrm{ccm} \mathrm{m}-\mathrm{KH}_{2} \mathrm{PO}_{4}, 0,2 \mathrm{ccm} 20 \%$ ige Fruktose, etwas Hexosephosphat und mit 200 fach Wasser gewaschenen Ultrafiltrationsrückstand $0,4 \mathrm{ccm}$.

(1) Rückstand 3 fach eingedickt gegenüber ursprünglichem Saft.

(2) Rückstand $11 / 2$ fach eingedickt.

(3) Rückstand 0,75 fach eingedickt.

$\mathrm{cmm} \mathrm{CO}_{2}$ Y 4 .

\begin{tabular}{|c|c|c|c|c|c|c|}
\hline & \multicolumn{2}{|c|}{ (1) } & \multicolumn{2}{|c|}{ (2) } & \multicolumn{2}{|r|}{ (3) } \\
\hline & $\begin{array}{l}3 \text { facher } \\
\text { Gesamt }\end{array}$ & $\begin{array}{l}\text { Rückstand } \\
\text { |pro15 Min. }\end{array}$ & $\begin{array}{l}1 / 2 \text { fach } \\
\text { Gesamt }\end{array}$ & $\begin{array}{l}\text { r Rückstd. } \\
\text { |pro 15 Min. }\end{array}$ & $\begin{array}{r}0,75 \text { fach } \\
\text { Gesamt }\end{array}$ & $\begin{array}{l}\text { er Ruckstd. } \\
\text { |pro15 Min. }\end{array}$ \\
\hline $18^{\prime}$ & 174 & 145 & 71 & 59 & 33 & 28 \\
\hline $30^{\prime}$ & 332 & 198 & 145 & 93 & 79 & 58 \\
\hline $45^{\prime}$ & 473 & 141 & 249 & 104 & 113 & 34 \\
\hline$\left(\begin{array}{ll}\text { h } & 15^{\prime}\end{array}\right)$ & (585) & 56 & (422) & 86 & (197) & 42 \\
\hline
\end{tabular}

Versuch 2. $1,3 \mathrm{ccm}$ Gärungsgemisch, enthaltend $0,6 \mathrm{ccm}$ Muskelkochsaft, $0,2 \mathrm{ccm} 20 \%$ ige Fruktose, $0,1 \mathrm{ccm} \mathrm{m}-\mathrm{KH}_{2} \mathrm{PO}_{4}$, etwas Hexosephosphat und $0,2 \mathrm{ccm}$ Rückstand, mit 150 fach Wasser gewaschen.

(1) 3 fach eingedickt, (2) 1 fach eingedickt.

$\mathrm{cmm} \mathrm{CO}_{2} \times 4$.

\begin{tabular}{|c|c|c|c|c|}
\hline & \multicolumn{2}{|c|}{ (1) } & \multicolumn{2}{|c|}{ (2) } \\
\hline & 3 fach konz. & Rückstand & 1 fach konz. & Rückstand \\
\hline & Gesamt & pro $15 \mathrm{Min}$. & Gesamt & pro $15 \mathrm{Min}$. \\
\hline $19^{\prime}$ & 83 & 65 & 15 & 12 \\
\hline $30^{\prime}$ & 230 & 198 & 59 & 60 \\
\hline $1 \mathrm{~h} 30^{\prime}$ & 595 & 91 & 232 & 43 \\
\hline$(2 \mathrm{~b} 15)$ & (714) & 40 & (333) & 33 \\
\hline
\end{tabular}

Versuch 3. Je $1,4 \mathrm{ccm}$ Gemisch, enthaltend $0,7 \mathrm{ccm}$ Hefekochsaft, $0,2 \mathrm{ccm} 20 \%$ ige Fruktose, etwas Hexosephosphat (kein äußeres Phosphat) und 150 fach gewaschenen Rückstand $0,4 \mathrm{ccm}$.

(1) Rückstand 3 fach konzentriert (gegenüber Ausgangssalt).

(2) Rückstand 1,5 fach konzentriert.

(3) Rückstand 0,5 fach konzentriert.

Außerdem (4) Rückstand 1,5 fach konz. und 0,35 ccm Hefekochsaft - statt $0,7 \mathrm{ccm}$. 
$\mathrm{cmm} \mathrm{CO} 2 \times 4$.

\begin{tabular}{|c|c|c|c|c|c|c|c|c|}
\hline & \multicolumn{2}{|c|}{ (1) } & \multicolumn{2}{|c|}{ (2) } & \multicolumn{2}{|c|}{ (3) } & \multicolumn{2}{|c|}{ (4) } \\
\hline & $\begin{array}{l}3 \text { fach. } \\
\text { Gesamt }\end{array}$ & $\begin{array}{l}\text { Rückst. } \\
\text { p.10 Min }\end{array}$ & $\begin{array}{l}11 / \mathrm{afac} \\
\text { Gesamt }\end{array}$ & $\begin{array}{l}\text { Rückst. } \\
.10 \mathrm{Min} \text {. }\end{array}$ & $\begin{array}{l}0,5 \text { fach } \\
\text { Gesamt }\end{array}$ & $\begin{array}{l}\text { Rückst. } \\
\text { |p.10Min. }\end{array}$ & $\begin{array}{c}11 / 2 \mathrm{fac} \\
1 / 2 \mathrm{~K} \\
\text { Gesam }\end{array}$ & $\begin{array}{l}\text { Rückst. } \\
\text { chsaft } \\
\text { p.10Min. }\end{array}$ \\
\hline $18^{\prime}$ & 239 & 133 & 118 & 65 & 25 & 14 & 105 & 58 \\
\hline $25^{\prime}$ & 371 & 189 & 205 & 124 & 74 & 70 & 180 & 107 \\
\hline$(35)$ & $(455)$ & 84 & (325) & 120 & (132) & 60 & (216) & 36 \\
\hline
\end{tabular}

Aus der Spalte 4 des letzten Versuchs ist zu sehen, daß bei Verringerung der Goenzymkonzentration trotz entsprechender gleichzeitiger Verringerung des Phosphats der Gäranstieg langsamer erfolgt. Wir sind also in einem Bereich, wo der Cofermentgehalt eine Rolle für die Gärungsanfangsgeschwindigkeit spielt!

IV. Beobachtungen zu den narkotischen Gärungshemmungen im Macerationssaft und Trockenhefe.

Im Anschluß an den kürzlich mitgeteilten Befund, ${ }^{1}$ ) daß die Fällungen im Macerationssaft durch Narkotika nur in Gegenwart von Salz entstehen, im gewaschenen Ultrafiltrationsrückstand, der aufs alte Volumen verdünnt ist, vollständig ausbleiben, anderseits bei Vermehrung des Salzgehalts, z. B. durch $\mathrm{NaCl}$, sehr verstärkt werden, untersuchen wir zunächst, ob eine ähnliche Abhängigkeit vom Salzgehalt auch für die Hemmung durch Narkotika besteht. Extreme Fälle können bei der zellfreien Gärung leider nicht untersucht werden, denn diese bedarf der Gegenwart von Salzen und des von Elektrolyten schwer abtrennbaren Coferments und verträgt anderseits keine beträchtliche Erhöhung der Salzkonzentration, da schon bei Zusatz von $1,5 \% \mathrm{NaCl}$ die Gärung im Macerationssaft stark gehemmt wird. Immerhin reicht die Variationsbreite aus, um festzustellen, daß tatsächlich Unterschiede in dieser Richtung bestehen und die Hemmungsgröße in Gegenwart von $\mathrm{NaCl}$ (relativ zur Gärung mit $\mathrm{NaCl}$, aber ohne Narkotikum)

1) Biochem. Zeitschr. (1918).

Hoppe-Seyler's Zeitschrift f. physiol. Chemie. CII. 
größer ist als ohne Kochsalzzusatz. Es ist dabei aber hervorzuheben, daß 1. die Gärung gegen Salz selbst sehr empfindlich ist, und diese Salzempfindlichkeit scheint sogar Bedingung für die Verstärkung der Wirkung des Narkotikums zu sein, und daß 2. nur solche Hemmungen, die schon ohne Salz deutlich sind, verstärkt werden, aber Stoffe, deren narkotischer Effekt nur gerade den Schwellenwert erreicht, etwa Phenylurethan in Sättigungskonzentration, auch mit $\mathrm{NaCl}$ zusammen nicht hemmen. Der Salzzusatz wirkt also anders als die $<$ Zellstruktur, in den Versuchen von Warburg, da diese die im Saft noch nicht wirksamen Narkotikumkonzentrationen zur Hemmung in der Zelle befähigt.

Diese Messungen können nicht in der «Phosphatperiodes gemacht werden, weil sich in dieser die Gärungsgeschwindigkeit zu rasch ändert. Daher wurde in der Regel der Saft zunächst mit Zucker einige Zeit in den Thermostaten gehängt, bis das Phosphat verbraucht war, die Kohlensäure fortgeschüttelt und dann gleiche Mengen Saft in die Gärungsgefäße gegeben und die Narkotika und Salzlösung hinzugesetzt. Wir bestimmen so die Hemmung der Hexosephosphatase, wie das schon früher von Dorner geschehen ist. ${ }^{1}$ ) Man kann sich jedoch davon überzeugen, daß dusselbe auch für die eigentliche Zymasereaktion gilt. Inkubiert man z. B. Saft mit Zucker und ziemlich viel Phosphat nur so lange, daß noch nicht alles Phosphat verbraucht, das Maximum aber schon überschritten ist, so kann man auch jetzt für eine kurze Zeit - etwa $15 \mathrm{Mi}-$ nuten - die gleichen Hemmungen feststellen. Und endlich gelingt das auch für die Anfangszeit, wenn man durch Zusatz von Arseniat für eine Konstanz der Gärungsgröße sorgt. Wenn man erst nach Ablauf der Phosphatperiode Arseniat hinzufügt, bekommt man zwar ohne Narkotika dieselben Werte, aber die Hemmungen sind dann unregelmäßig, meist größer.

Die Zusammensetzung der Gärungsgemische in den folgenden Versuchen war ähnlich der bisherigen; Flüssigkeitsmenge meist $2 \mathrm{ccm}$. Innerhalb jeder Versuchsserie waren alle Abmessungen genau gleich bis auf die NaCl- und Narkotikumkonzentrationen.

1) Diese Zeitschrift, Bd. 81, S. 99 (1912). 
I. Inkubation des Hefesaftes mit Zucker (ohne Phosphat) während 1-2 Stunden. Darauf die folgenden Messungen.

\begin{tabular}{|c|c|c|c|c|c|c|c|}
\hline & Zeit & Narkotikum & $\left|\begin{array}{c}\text { ohne } \\
\mathrm{cmm} \\
\mathrm{CO}_{2} \times 4\end{array}\right|$ & $\begin{array}{l}\mathrm{NaCl} \\
\mathrm{Hem}- \\
\text { mung } \\
\text { in } \%\end{array}$ & $\begin{array}{r}r \\
\% \\
\mathrm{NaCl}\end{array}$ & $\begin{array}{l}\text { mit } \mathrm{NaC} \\
\mid \begin{array}{c}\mathrm{cmm}^{2} \\
\mathrm{CO}_{\mathbf{2}} \times 4\end{array}\end{array}$ & $\begin{array}{l}1 \\
\text { Hem- } \\
\text { mung } \\
\text { in } \%\end{array}$ \\
\hline 1: & $50^{\prime}$ & $6 \%$ Äthylurethan & $\begin{array}{l}138 \\
111\end{array}$ & 20 & $1,2 \%$ & $\begin{array}{r}119 \\
76\end{array}$ & 35 \\
\hline 2. & $45^{\prime}$ & $6 \%$ Äthylurethan & $\begin{array}{l}167 \\
129\end{array}$ & 23 & $1,35 \%$ & $\begin{array}{l}96 \\
52\end{array}$ & 46 \\
\hline 3. & $45^{\prime}$ & $6 \%$ Äthylurethan & $\begin{array}{l}275 \\
183\end{array}$ & 33 & $1,3 \%$ & $\begin{array}{r}158 \\
80\end{array}$ & 49 \\
\hline 4. & $30^{\prime}$ & $\begin{array}{l}40 \% \text { Äthylurethan } \\
1 \% \mathrm{i} \text {-Butylurethan } \\
\text { gesätt. Amylalkohol }\end{array}$ & $\begin{array}{r}211 \\
169 \\
164 \\
28\end{array}$ & $\begin{array}{l}20 \\
22 \\
87\end{array}$ & $1,2 \%$ & $\begin{array}{r}145 \\
102 \\
103 \\
0\end{array}$ & $\begin{array}{r}30 \\
30 \\
100\end{array}$ \\
\hline 5. & $30^{\prime}$ & $\begin{array}{l}1 \% \text { i-Butylurethan } \\
\text { ges. Phenylurethan } \\
\text { ges. Heptylalkohol }\end{array}$ & $\begin{array}{l}184 \\
147 \\
182 \\
154\end{array}$ & $\begin{array}{r}20 \\
0 \\
16\end{array}$ & $1,2 \%$ & $\begin{array}{r}123 \\
89 \\
118 \\
106\end{array}$ & $\begin{array}{r}28 \\
4 \\
14\end{array}$ \\
\hline 6. & $30^{\prime}$ & $\begin{array}{l}10 \% \text { Methylurethan } \\
3 \% \text { Propylurethan }\end{array}$ & $\begin{array}{r}102 \\
48 \\
68\end{array}$ & $\begin{array}{l}\mathbf{5 3} \\
\mathbf{3 3}\end{array}$ & $1,2 \%$ & $\begin{array}{l}71 \\
23 \\
36\end{array}$ & $\begin{array}{l}68 \\
50\end{array}$ \\
\hline
\end{tabular}

II. Inkubation mit Zucker und Phosphat $(\mathrm{m} / \mathrm{10})$ für $1 \mathrm{~h} 50^{\prime}$. Vor Ablauf der Phosphatperiode gemessen.
7. $\left|15^{\prime}\right|$
$6 \%$ Äthylurethan
99
24 \begin{tabular}{l|l|l}
$0,9 \%$ & 81 & \\
& 41 & 50
\end{tabular}

III. Gemisch: $1,2 \mathrm{ccm}: 0,4 \mathrm{ccm}$ Saft $+0,2$ Fruktose $20 \%$; mit $0,005 \mathrm{~m}$ Arseniat. Messung nach Erreichung des Gärungsmaximums (30 Min. nach Vermischung und Zusatz der Narkotika).
8. ${ }^{30^{\prime}} \mid$
$4 \%$ Äthylurethạn

295
236
293
261
\begin{tabular}{r|}
20 \\
0 \\
12
\end{tabular}
$\mid 1,2 \%$

\begin{tabular}{r|r}
158 & \\
76 & 53 \\
156 & 0 \\
135 & 15
\end{tabular}

Auch frühere Beobachtungen von mir dürften im Sinne einer Abhängigkeit der Hemmungsstärke der Narkotika von 
der Salzkonzentration $z u$ deuten sein. So wurde gefunden, ${ }^{1}$ ) daß die Atmungshemmung im Brei von Seeigeleiern, der durch Zerschütteln unbefruchteter Eier mit dem gleichen Volumen destillierten Wassers gewonnen war, im Anfang ziemlich gering war und erst im Lauf von $1-11 / 2$ Stunden stärker zunahm, ohne die Hemmung lebender Eier zu erreichen. Wurde dagegen das Narkotikum zu der Suspension intakter Eier hinzugefügt und dann erst die Zerschüttelung vorgenommen, so war die Hemmung stärker und ziemlich konstant. Im ersteren Fall wirken die Narkotika in salzärmerem Milieu auf die Eisubstanz ein. - Anderseits scheint eine Salzempfindlichkeit des zu hemmenden Enzyms selbst erforderlich zu sein, damit eine solche Abhängigkeit der narkotischen Wirkungsstärke vom Salzgehalt in Erscheinung tritt. Obgleich nämlich die Atmung des Hefemacerationssafts von etwa denselben Narkotikumkonzentrationen gehemmt wird wie die Gärung ${ }^{2}$ ) - eher noch etwas stärker - , so wird diese Atmungshemmung nicht einmal in Gegenwart von $5 \% \mathrm{NaCl}$ verstärkt, obwohl der Unterschied der Fällungsstärke der Narkotika in diesem Fall beträchtlich ist. Aber auch die Atmungsgröße selbst wird durch den Salzzusatz nicht geändert. Es ist eben daran zu denken, daß die Fällungen, obwohl sie ja zweifellos mit derselben Eigenschaft der Narkotika in Zusammenhang stehen, die auch für die Hemmung wesentlich ist, nämlich der Oberflächenaktivität, doch nicht unmittelbar die Erklärung der Wirkung zu geben brauchen. Hierfür genügt, wie ich schon öfters hervorhob, die Verdrängung des Substrats von der kolloiden Enzymoberfläche, die auch ohne sichtbare Dispersitätsänderung von statten gehen kann, aber allerdings bei den Stoffwechselfermenten meist damit verbunden ist. . In welchem Umfang diese Dispersitätsverringerung für die Größe der Hemmungen eine Rolle spielt, ist noch eine offene Frage, zu deren Beantwortung die vorstehenden Versuche nur einen Beitrag liefern.

$\mathrm{Da}$ in den mitgeteilten Experimenten mit Inkubation des zuckerhaltigen Hefesafts die Hemmung der Hexosephos-

1) Pflügers Archiv, Bd. 157, S. 287 (1914).

2) Pflügers Archiv, Bd. 170, (1918). 
phatase geprüft wird, so muß man offenbar dasselbe Resultat erhalten, wenn man die Gärung des Hexosephosphats (ohne Inkubation) - durch Narkotika zu hemmen sucht. Wie oben gezeigt ist, ist diese Gärgeschwindigkeit von vorne herein ziemlich konstant und wird allein durch die Tätigkeit des esterspaltenden Ferments bestimmt. Das steht nun in Widerspruch mit dem von Euler und Johansson aus ihren Experimenten an Trockenhefe gezogenen Schluß: ‘Es ergibt sich also als allgemeines Resultat, daß die enzymatische Spaltung des Kohlehydratphosphorsäureesters durch antiseptische Mittel wie Toluol stark gehemmt wird *.1) Tatsächlich wird aber in Übereinstimmung mit unsern obigen Befunden die Vergärung des Hexosephosphats im Macerationssaft - also auch die enzymatische Spaltung des Esters - durch Toluol in Sättigungskonzentration, ebenso durch Phenylurethan oder durch Heptylalkohol in Sättigungskonzentration gar nicht oder nur äußerst geringfügig gehemmt, und auch die übrigen Hemmungen stimmen genau mit den obigen überein. Das Resultat der schwedischen Autoren kommt offenbar dadurch zustande, daß sie ihre Versuche mit Trockenhefe anstellten, also keine Enzymlösung vor sich hatten. In der Tat ist die Gärungshemmung in Trockenhefe erheblich größer als im Hefesaft und liegt zwischen der Safthemmung und der Zellhemmung, auch insofern, als die Verstärkung vor allem die höheren Glieder der homologen Reihen betrifft. In den folgenden Versuchen wird die Vergärung des Hexosephosphats durch Aufschwemmungen von Trockenhefe unter der Wirkung derselben Narkotikumkonzentrationen geprüft, die bei den Safthemmungen zur Verwendung kamen. Die Suspensionen von Trockenhefe waren doppelt verdünnt gegenüber den zur Herstellung des Macerationssafts dienenden, in Menge von $1,2-1,6 \mathrm{ccm}$.

Diese Hemmungen, die übrigens ähnlich den Hemmungen im Saft progressiv sind, kann man nicht dahin verstehen, daß sie die Resultante von Zellhemmungen und Safthemmungen vorstellen, indem man die Trockenhefe als ein Gemisch von teils lebenden, teils abgestorbenen Zellen auffaßt. Zugesetztes

1) Diese Zeitschr., Bd. 85, S. 204 (1913). 


\begin{tabular}{|c|c|c|c|c|}
\hline & Zeit & Narkotikum & $\frac{\mathrm{cmm}}{\mathrm{CO}_{2} \times 4}$ & $\begin{array}{l}\text { Hemmung } \\
\text { in } \%\end{array}$ \\
\hline 1. & $50^{\prime}$ & gesättigt Toluol ') & $\begin{array}{l}328 \\
233\end{array}$ & 30 \\
\hline 2. & $30^{\prime}$ & $\begin{array}{l}6 \% \text { Äthylurethan } \\
1 \% \text { i-Butylurethan }\end{array}$ & $\begin{array}{r}106 \\
57 \\
58\end{array}$ & $\begin{array}{l}46 \\
45\end{array}$ \\
\hline 3. & $40^{\prime}$ & $\begin{array}{c}6 \% \text { Äthylurethan } \\
1 \% \text { i-Butylurethan } \\
\text { gesätt. Phenylurethan }\end{array}$ & $\begin{array}{l}217 \\
104 \\
130 \\
112\end{array}$ & $\begin{array}{l}52 \\
40 \\
48\end{array}$ \\
\hline
\end{tabular}

Hexosephosphat wird von lebenden Zellen überhaupt nicht gespalten, weil es die Zellwand nicht durchdringt.") Nur die abgetöteten Zellen nehmen also an der Esterspaltung teil und auch hier ist diese Reaktion die langsamst verlaufende des Gärungsprozesses: denn durch Arseniat wird auch die Hexosephosphatvergärung der Trockenhefe mächtig gesteigert. Die gemessenen Hemmungen betreffen also in der Tat die in den abgetöteten Zellen vorhandene Hexosephosphatase.

Nur die Wirkung des Heptylalkohols (in gesättigter Lösung) gegenüber der Hexosephosphatvergärung durch Trockenhefe weicht völlig von seinem sonstigen Verhalten ab. Dieser hemmt nicht nur nicht, sondern steigert diesen Prozeß um 50-80\%. Die Ursache hiervon ist ganz unklar. Denn weder hydrolysiert der Heptylalkohol für sich das Hexosephosphat, noch zeigt sich dieser Effekt in merklichem Grade (höchstens angedeutet) bei der Hexosephosphatvergärung im Macerationssaft. Und auch die Fruktosegärung durch Trockenhefe nach längerer Inkubation mit Zucker - also im Stadium der Hexosephosphatspaltung wird nicht gesteigert, sondern gehemmt (etwa 25\% in Sättigungskonzentration). Vielleicht kann man diesen Befund in

1) Bei Versuchen mit Überschuß von Toluol müssen die Gärungsgefäße anfangs so lange geschüttelt werden, bis der durch Verdunstung des Toluols entstandene. Druck konstant bleibt. Dieser Wert $(67 \mathrm{~mm}$ Manometerdruck) wird in Abzug gebracht.

- $\quad$ 2) Paine, Proc. Roy. Soc. B., Bd. 84, S. 304 (1912). 
Analogie setzen mit der Feststellung von Harden und $\left.\mathrm{Paine},{ }^{1}\right)$ daß Toluol und höhere Konzentrationen Äthylalkohol die Selbstgärung lebender Hefe steigern und durch eine Vermehrung der Durchlässigkeit der Trockenhefezellen für Hexosephosphat unter dem Einfluß des Heptylalkohols erklären. Jedoch zeigen andere, sonst ähnlich wirkende Stoffe, z. B. Toluol oder Amylalkohol, das Phänomen nicht.

\begin{tabular}{|c|c|c|c|c|}
\hline & Zeit & ohne Hept. & $\begin{array}{l}\mathrm{mm} \mathrm{CO}_{2} \times \\
\text { mit Hept. } \\
\text { gesättigt }\end{array}$ & $\begin{array}{l}4 \\
\substack{\text { Steigerung } \\
\%}\end{array}$ \\
\hline $\begin{array}{l}\text { 1. } 2,4 \mathrm{ccm} \text { Gemisch : } 1,4 \mathrm{ccm} \\
\text { Hefesusp., } 0,02 \mathrm{~m} \text {-Hexose- } \\
\text { phosphat. } . . . . . .\end{array}$ & $40^{\prime}$ & 217 & 319 & 45 \\
\hline $\begin{array}{l}\text { 2. } 1,8 \mathrm{ccm} \text { Gemisch : } 1,4 \mathrm{ccm} \\
\text { Hefesusp., } 0,03 \mathrm{~m} \text {-Hexose- } \\
\text { phosphat. . . . . . . }\end{array}$ & $75^{\prime}$ & 270 & 492 & 80 \\
\hline $\begin{array}{l}\text { 3. } 1,6 \mathrm{ccm} \text { Gemisch }: 1,0 \text { Trock.- } \\
\text { Hefesusp., } 0,018 \mathrm{~m} \text {-Hexose- } \\
\text { phosphat. . . . . . . }\end{array}$ & $25^{\prime}$ & 92 & 143 & 55 \\
\hline $\begin{array}{l}\text { 4. } 1,6 \mathrm{ccm} \text { Gemisch : } 1,0 \text { Trock.- } \\
\text { Hefesusp., } 0,05 \mathrm{~m} \text {-Hexose- } \\
\text { phosphat. . . . . . . }\end{array}$ & $100^{\prime}$ & 270 & 487 & 80 \\
\hline
\end{tabular}

Auf ein anderes Faktum sei in diesem Zusammenhange noch hingewiesen. Während Hexosephosphat von lebender Hefe nicht vergoren wird, war die Vergärung durch Trockenhefe um den fünffachen Betrag größer als in dem aus ihr gewonnenen, allerdings nicht sehr gärkräftigen Saft unter den gleichen Konzentrationsbedingungen. Gerade die schlechte Extraktionsfähigkeit der Hexosephosphatase bezw. ihr Wirksamkeitsabfall beim Auslösen aus der Zelle sind ja der eigentliche Grund dafür, daß die Gärgeschwindigkeit in vitro so weit hinter der Zellgärung zurückbleibt. Die Zymase selbst und das Coferment lassen sich so gut extrahieren, daß die Gärgeschwindigkeit annähernd der der lebenden Zellen gleichkommt, wenn man durch Arseniat die Esterspaltung genügend beschleunigt.

1) Proc. Roy. Soc. B.., Bd. 84, S. 448 (1912). 


\section{Zusammenfassung.}

1. Bei mangelnder Selbstgärung kann vor dem Beginn der Gärung des Macerationssafts nach Zuckerzusatz ein längeres oder kürzeres Intervall liegen: die «Induktion». Verschiedene Umstände beeinflussen seine Länge: Bemerkenswert erscheint u. a., daß - ceteris paribus - Saccharose viel schneller angegoren wird wie Fruktose und Glukose, dab letztere nach längerem Erwärmen mit Phosphat schneller angegoren werden, daB die Induktion durch Zerreiben der Trockenhefe mit Glaspulver sehr verkürzt wird, daß sie durch Hexosephosphat von 0,2 Millimol an mit Sicherheit momentan aufgehoben wird.

2. Die Gärung zeigt eine starke Anfangsbeschleunigung, herrührend von im Saft vorhandenem freien Phosphat (Harden). Das Maximum der Gärgeschwindigkeit wird aber nicht sofort erreicht. Der Anstieg der Geschwindigkeit erfolgt um so langsamer, je höher die Anfangskonzentration des Phosphats ist, während dabei die Maxima zunächst noch etwas steigen, dann aber auch fallen. Ähnlich wie Erhöhung des Phosphatsgehalts wirkt Zusatz eines anderen Salzes, z. B. NaCl, sowohl auf die Verlangsamung des Gäranstiegs wie auf die Herabsetzung des Geschwindigkeitsmaximums. Neben der spezifischen Funktion hat das Phosphat noch eine allgemeine «Salzfunktion », durch die es von mäßiger Konzentration an die Gärgeschwindigkeit hemmt.

3. Umgekehrt erfolgt der Gäranstieg mit steigender Konzentration des zugesetzten Hexosephosphorsäureesters immer schneller, wobei es auf diesen selbst, nicht auf die aus ihm abgespaltene Hexose ankommt. Das Phänomen des Gäranstiegs wird nicht vollständig durch die autokatalytische Wirkung des gebildeten Phosphorsäureesters in Verbindung mit der «negativ katalytischen , des verschwindenden Phosphats erklärt; wahrscheinlich kommt hierzu noch eine zeitliche Dissoziation zwischen Phosphatveresterung und Kohlensäurebildung (Euler).

4. Der Gäranstieg erfolgt rascher bei zunehmendem Gehalt an Coferment. Für die Gärgeschwindigkeit ist maßgebend die.Konzentration des Coferments, nicht seine Menge im 
Verhältnis zur Zymasemenge. Dies spricht etwas gegen eine chemische Beteiligung des Körpers am Gärungsvorgang.

5. Die narkotischen Gärungshemmungen im Macerationssaft werden in Parellelismus zu den Fällungen durch $\mathrm{NaCl}-$ Zusatz etwas verstärkt. Dies entspricht aber nicht der Wirkung der Zellstruktur (Warburg) auf die HemmungsgröBe. Die Hemmungen der Zymase und Hexosephosphatase im Saft sind gleich, dagegen wird die Vergärung des Hexosephosphats durch Trockenhefe schon von kleineren Konzentrationen gehemmt, die zwischen den für Saft und lebende Zellen geltenden Werten liegen. 